RESEARCH ARTICLE

\title{
Effectiveness of senior secondary schools in Sri Lanka
}

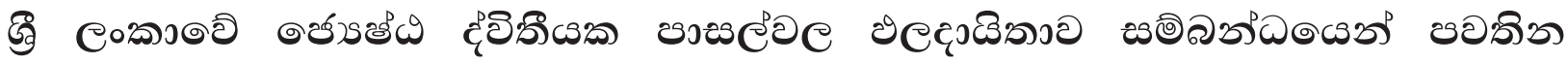 \\ ชิชอฺง
}

\author{
A M Hettige \\ Department of Social Science Education, Faculty of Education, University of Colombo, Colombo 03
}

\begin{abstract}
It is widely accepted that, in spite of the availability of a national policy, common educational objectives, curricula, text books, evaluation and examination systems to guide the general education process in Sri Lanka, there are clear inequalities within the system in terms of quality and effectiveness. The main reason for this diversity is that there are various factors affecting school effectiveness. It is difficult to provide a precise definition of, or statistical data, on school effectiveness based on the findings of studies done in different countries on the subject. Yet, it is possible to identify factors that determine effectiveness of schools in a particular country or area.
\end{abstract}

The main objective of the present study is to examine the factors that affect effectiveness of senior secondary schools in Sri Lanka. In order to achieve this objective, an exploratory research design involving sample surveys and case studies was employed. While questionnaires, observations, interviews and documentary analysis have been used as techniques of data collection, statistical methods were used for data analysis. The study was conducted in two stages in order to facilitate the use of both quantitative and qualitative approaches. Quantitative data was collected in stage one, while in stage two, case studies of six schools were undertaken in order to collect qualitative data. In the first stage of the study, 36 schools were drawn on the basis of a stratified random sample representing senior secondary schools in all educational divisions in the Colombo district. Then a sample of 500 stakeholders was drawn from these schools. The data collected from these respondents have been analyzed and interpreted in keeping with the objectives of the study.
Firstly, the researcher, based on the conceptual framework developed on the basis of the literature survey, formulated a questionnaire covering twelve factors and 95 criteria suitable to measure school effectiveness. Based on observations and questionnaires administered among stakeholders, the extent of the presence of school effectiveness factors were estimated. It was found that the principal's leadership, school mission and objectives, school culture and environment, curricula, student centeredness, and teachers' commitment were satisfactory in the sample schools. Yet, the situation with regard to school-community relations, student - teacher welfare, extent of extra - curricular activities and student expectations in schools and physical resources were not satisfactory. The factors that contribute to school effectiveness in the country as identified by stakeholders in descending order of importance are; school mission and objectives, school culture, principal's leadership, teacher commitment, student expectations, studentcenteredness of the curriculum, adequacy of resources and community relations.

A clear relationship was found between factors of school effectiveness and GCE Ordinary Level results. The weakest relationship was found between factors of school effectiveness and Grade Five scholarship examination results.

A school effectiveness index was developed and used to rank schools in terms of school effectiveness. Accordingly, schools were divided into three levelsranging from the highest to the lowest level of effectiveness. 
The above classification helped explain disparities among schools in terms of zone, school category, type of administration, composition and location. Accordingly, the greatest disparities were found in the Colombo division, while the least disparities were found in the Homagama division. As regards the type of schools, 9 out of 10 highest ranking schools belonged to $1 \mathrm{AB}$ (Schools with GCE A/L Science, Commerce and Arts classes) category. In other words, $1 \mathrm{AB}$ schools were more effective than 1C (Schools with GCE A/L Arts and Commerce classes) schools. However, among $1 \mathrm{AB}$ schools, national schools were higher in rank than provincial schools and private schools were higher in rank in comparison to both national and provincial schools. Among the government schools, national schools ranked higher than provincial schools and private schools ranked higher than government schools. As for student composition, male schools ranked higher than mixed schools. Yet, female schools ranked higher than male schools. Among mixed schools there are still disparities between $1 \mathrm{AB}$ and $1 \mathrm{C}$ schools, the former ranking higher. As for the location, disparities were greater among schools in Municipal areas than elsewhere.

In-depth analysis carried out confirmed the earlier findings as regards the factors determining school effectiveness. Moreover, in-depth data also confirmed the validity of the school effectiveness index developed on the basis of the quantitative survey. Accordingly, the conclusions derived from the study could be generalized with respect to schools in other parts of Sri Lanka.

\section{ఒ)రJoGacs}

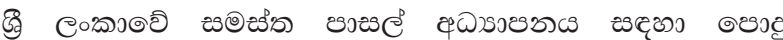

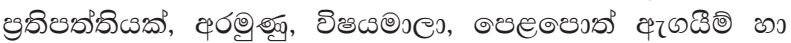

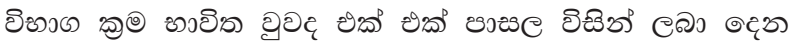

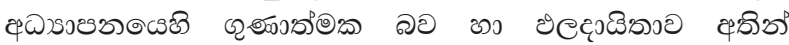

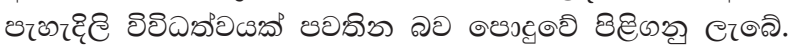

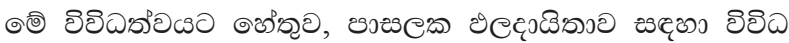

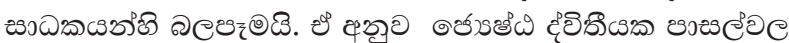

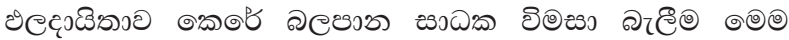

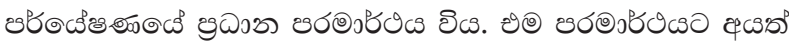

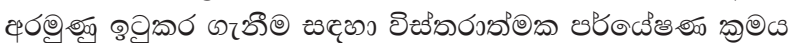

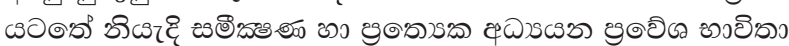

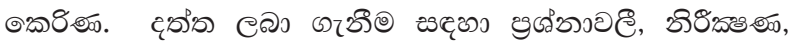

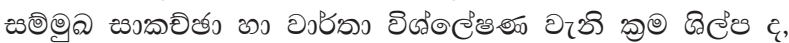

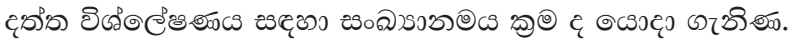

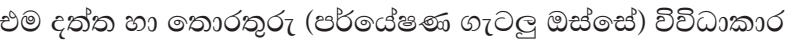

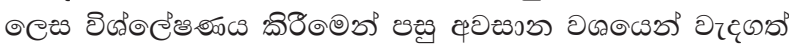

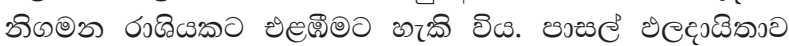

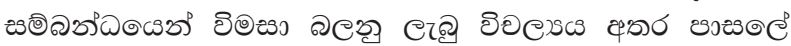

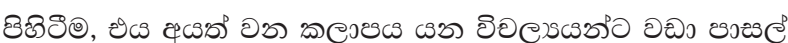

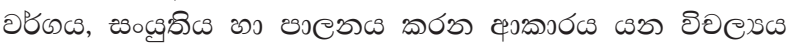

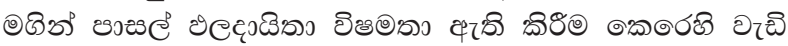

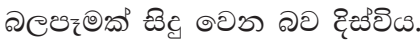

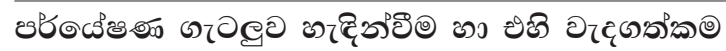

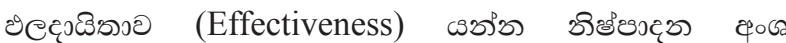

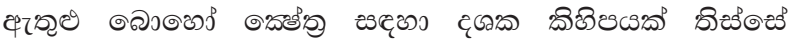

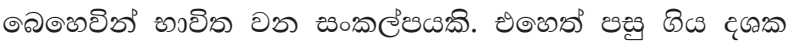

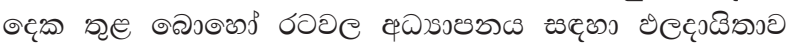

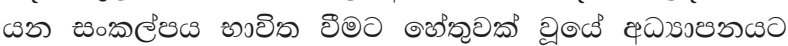

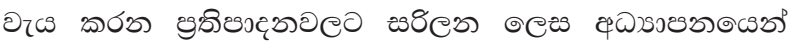

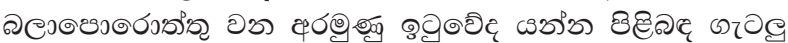

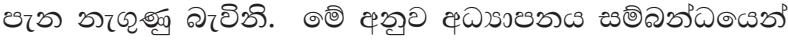

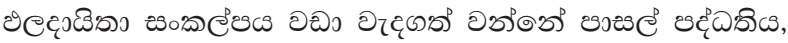

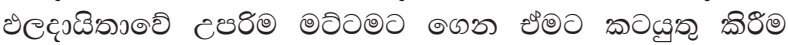

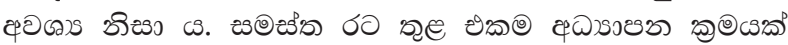

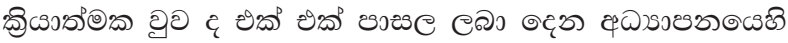

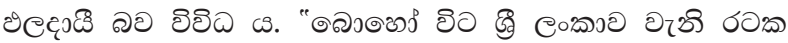

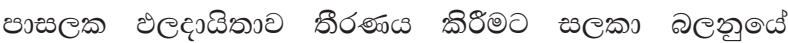

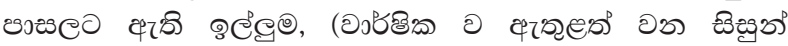

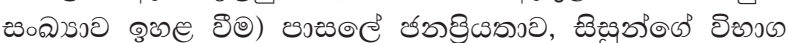

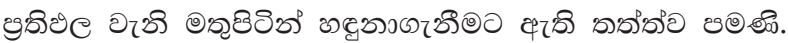

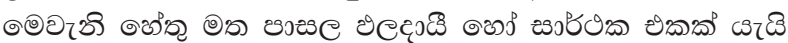

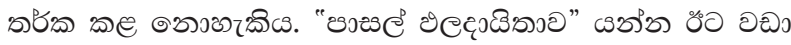

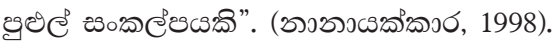

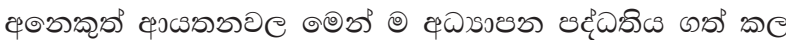

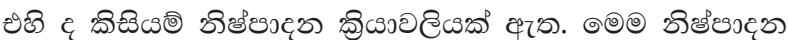

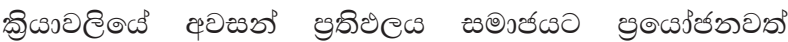

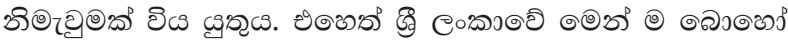

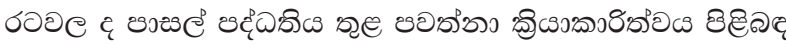

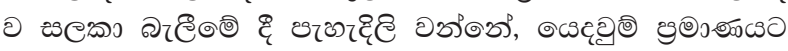

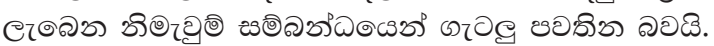

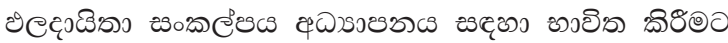

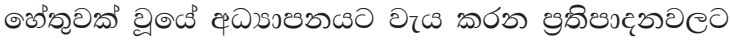

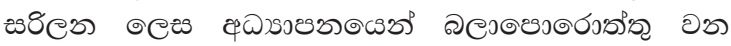

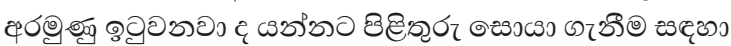

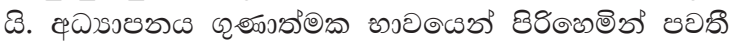

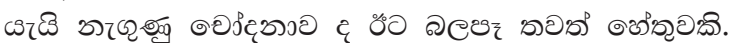

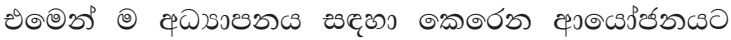

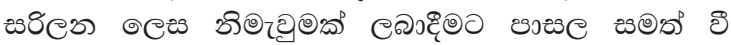

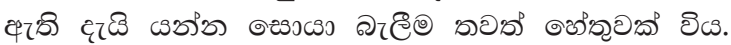

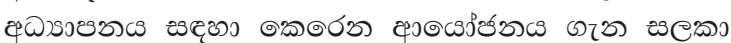

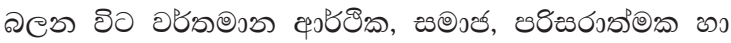

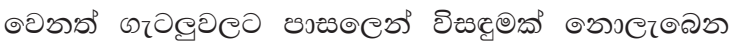

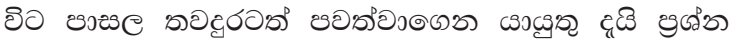
๑๐๑์. ( Mellor \& Chapman 1984)

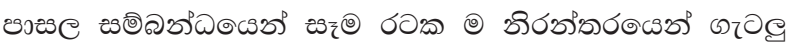




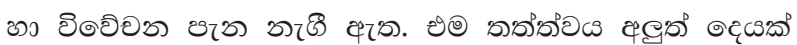

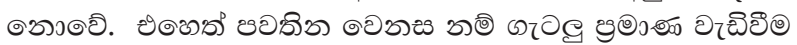

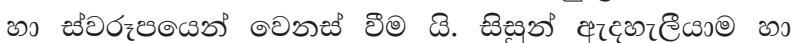

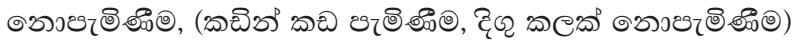

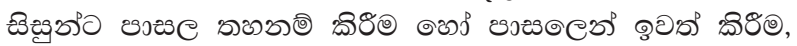

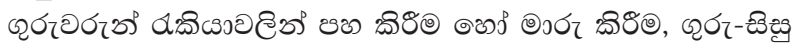

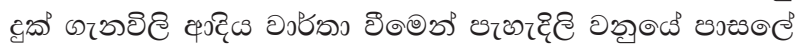

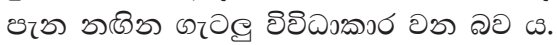

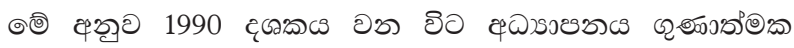

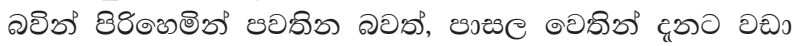

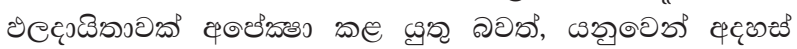

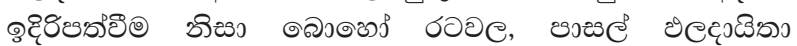

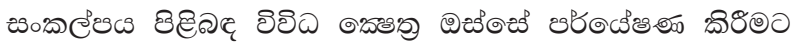

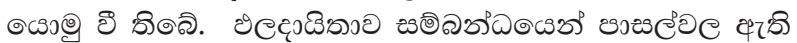

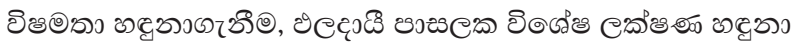

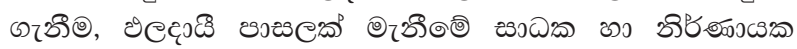

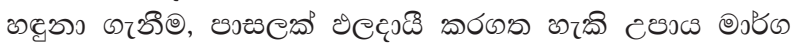

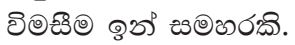

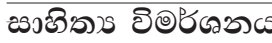

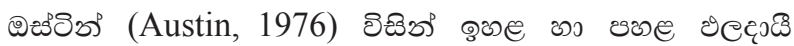

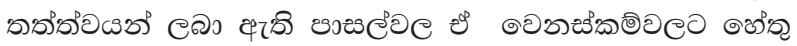

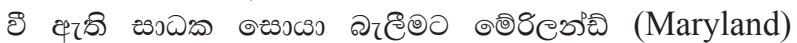

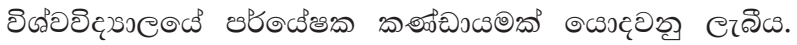

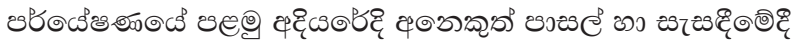

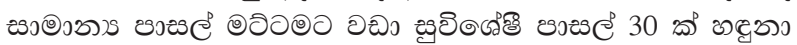

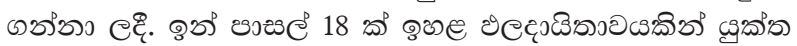

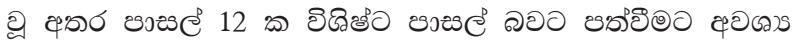

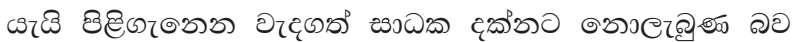

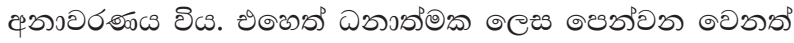

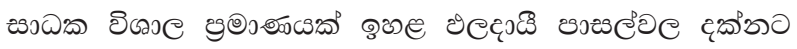

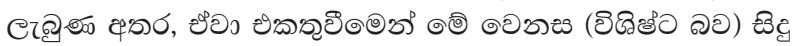

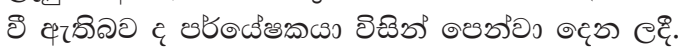

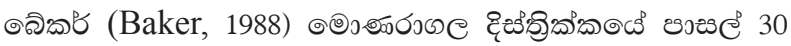

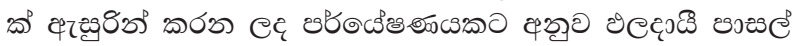

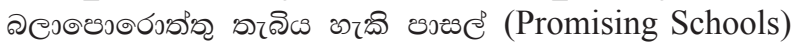

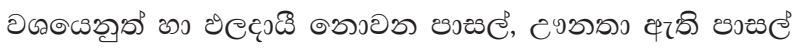

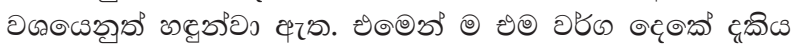

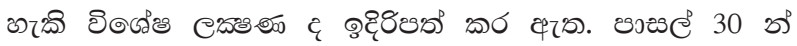

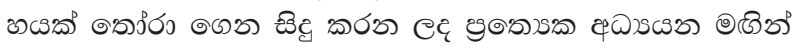

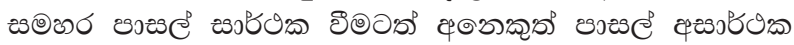

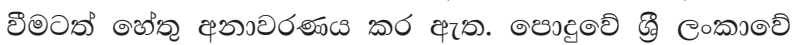

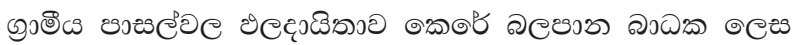

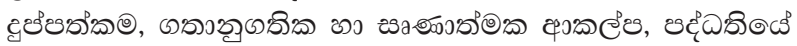

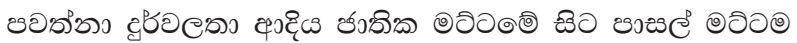

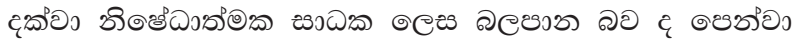

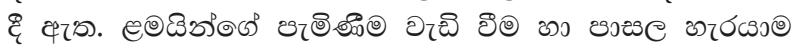

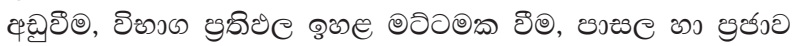

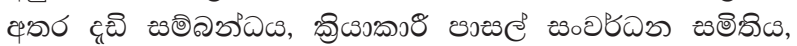

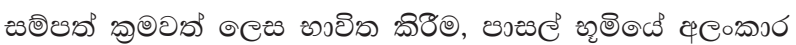

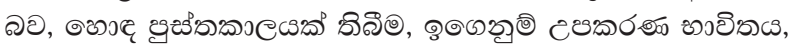

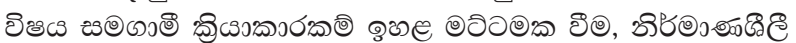

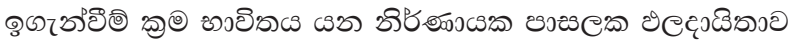

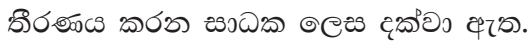

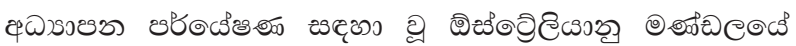
(Australian Council for Educational Research)

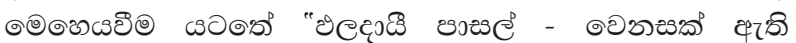

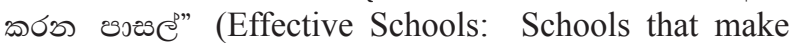

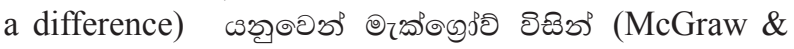

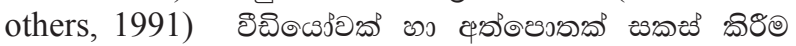

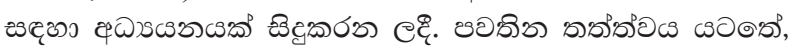

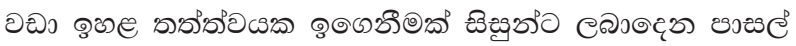

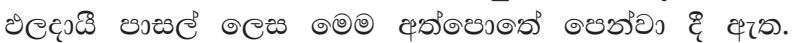

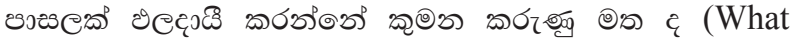

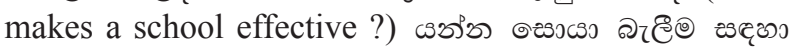

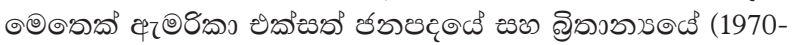

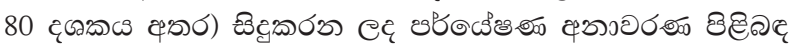

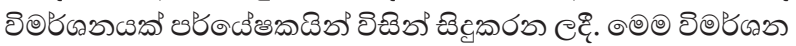

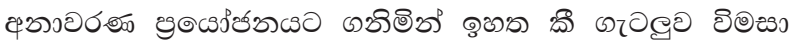

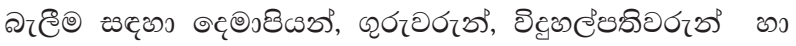

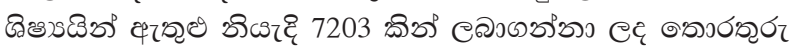

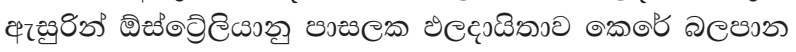

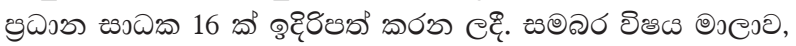

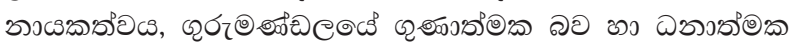

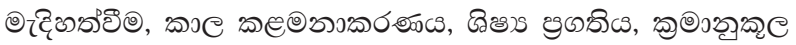

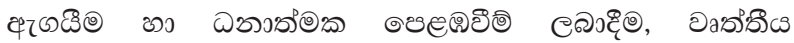

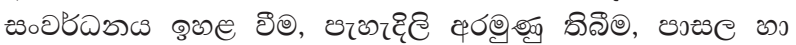

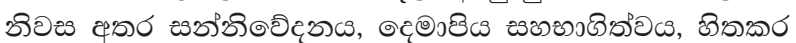

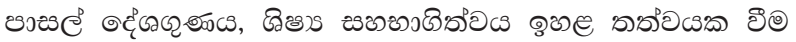

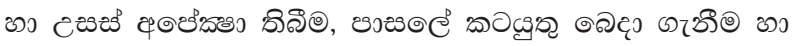

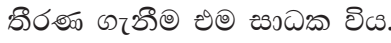

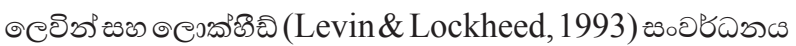

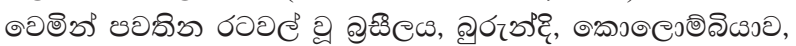

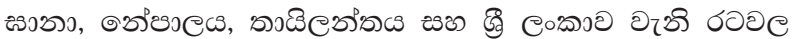

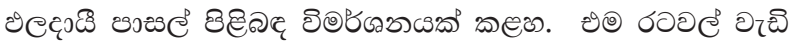

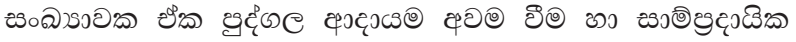

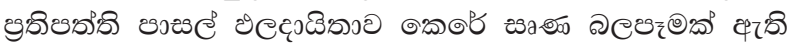

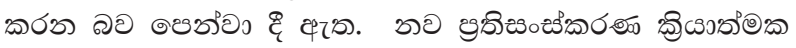

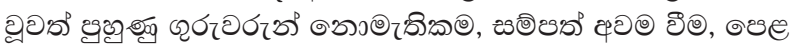

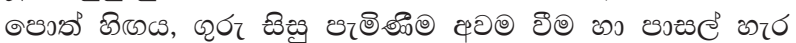

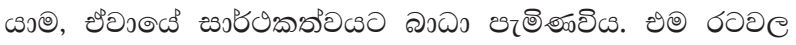

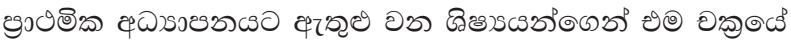

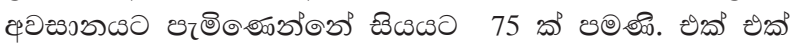

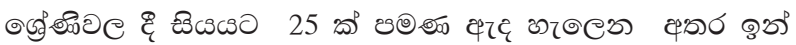

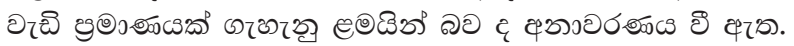

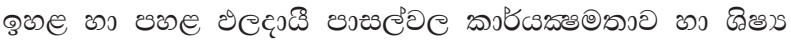

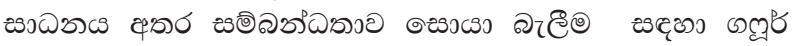

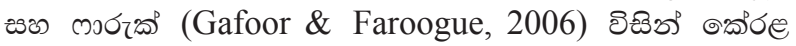

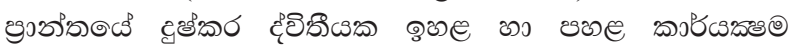




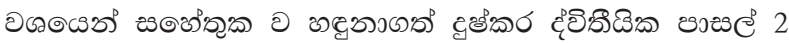

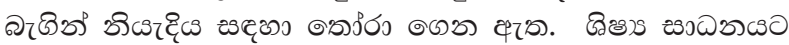
๑

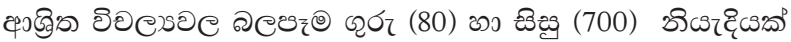

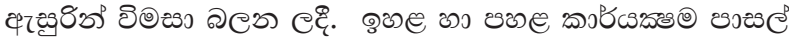

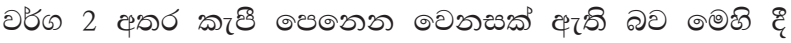

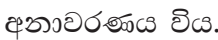

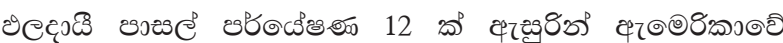

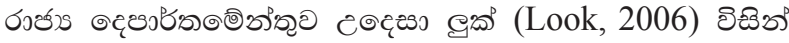

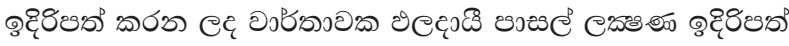

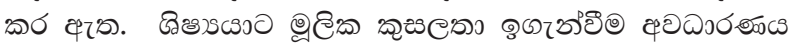

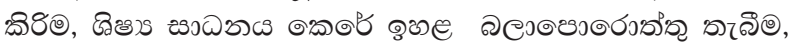

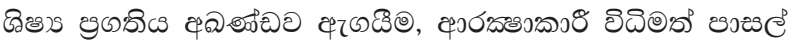

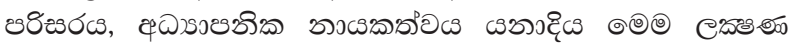

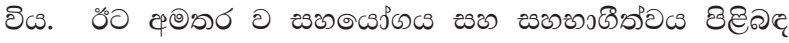

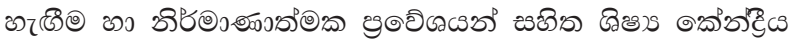

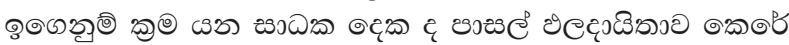

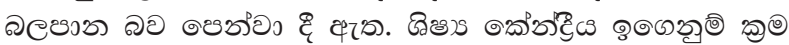

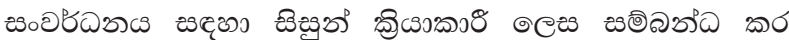

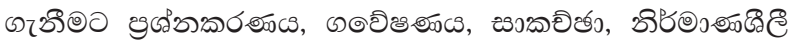

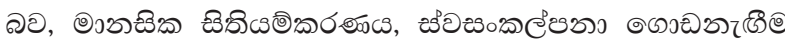

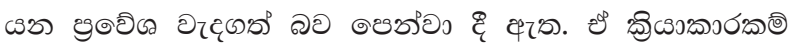

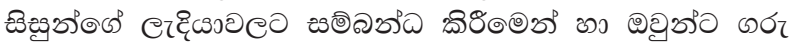

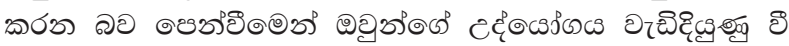

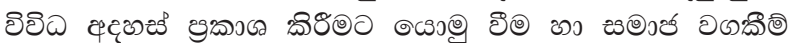

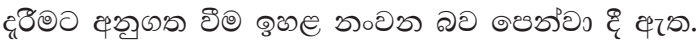

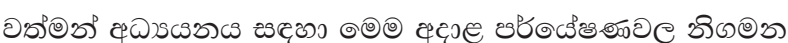
ల

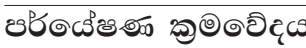

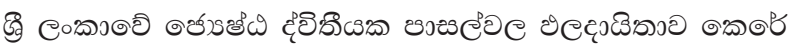

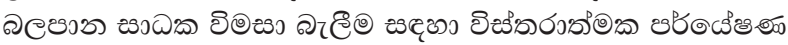

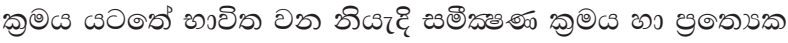

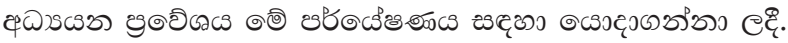

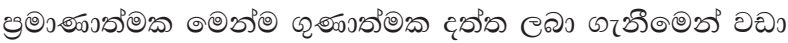

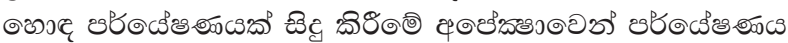

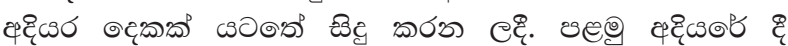

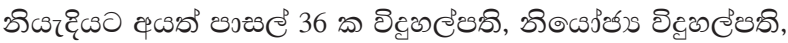

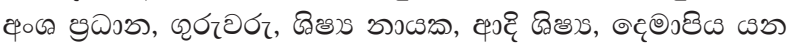

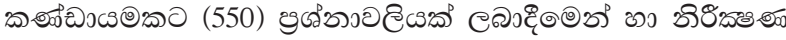

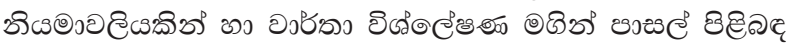

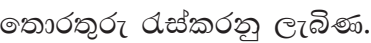

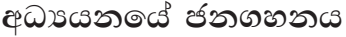

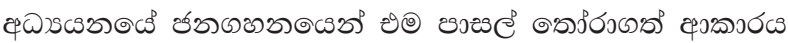

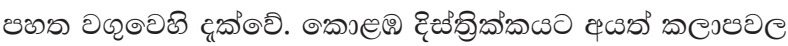

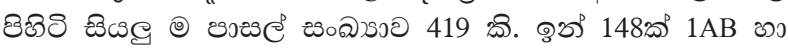

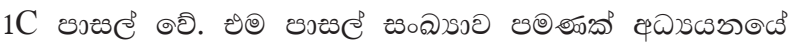

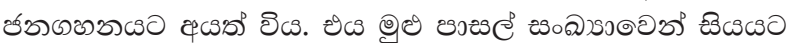

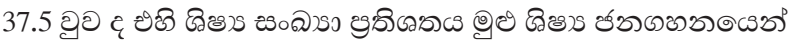

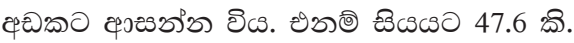

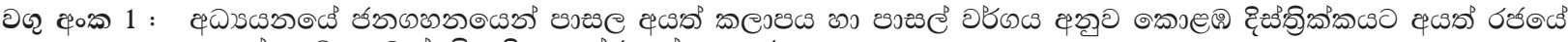

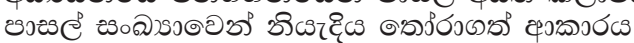

\begin{tabular}{|c|c|c|c|c|c|c|c|c|c|c|c|c|}
\hline \multirow[t]{3}{*}{ 2கejes } & \multirow{3}{*}{ 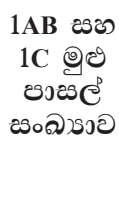 } & \multirow{3}{*}{ 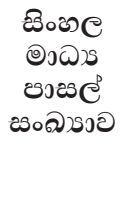 } & \multicolumn{4}{|c|}{ 1AB ঋڤ 1C ওృజల జంఎకృอ } & \multicolumn{4}{|c|}{ 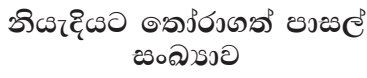 } & \multirow{2}{*}{\multicolumn{2}{|c|}{ ขึฌి }} \\
\hline & & & \multicolumn{2}{|c|}{$1 \mathrm{AB}$} & \multicolumn{2}{|c|}{$1 \mathrm{C}$} & \multicolumn{2}{|c|}{$1 \mathrm{AB}$} & \multicolumn{2}{|c|}{$1 \mathrm{C}$} & & \\
\hline & & & 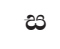 & э) & ¿s & 3 & జ & 3 & ¿s & త్ర & ఓి & э્) \\
\hline ๑றைீఱ & 57 & 41 & 22 & 5.0 & 19 & 4.4 & 5 & 16.6 & 4 & 13.3 & 9 & 30.0 \\
\hline 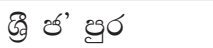 & 40 & 40 & 19 & 4.4 & 21 & 4.8 & 4 & 13.3 & 5 & 16.6 & 9 & 30.0 \\
\hline 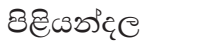 & 32 & 31 & 14 & 3.2 & 17 & 3.8 & 3 & 10.0 & 4 & 13.3 & 7 & 23.3 \\
\hline 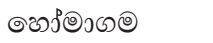 & 19 & 19 & 07 & 1.6 & 12 & 2.7 & 2 & 6.6 & 3 & 10.0 & 5 & 16.6 \\
\hline తివువు & 148 & 131 & 62 & 14.2 & 69 & 15.7 & 14 & 46.6 & 16 & 53.3 & 30 & 99.9 \\
\hline
\end{tabular}

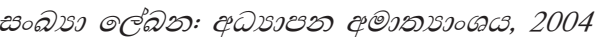




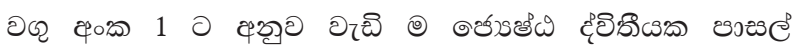

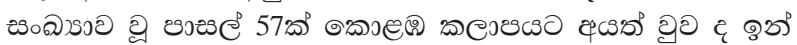

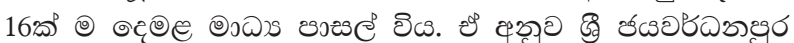

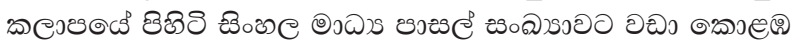

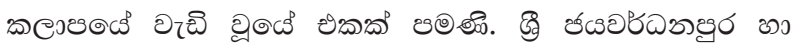

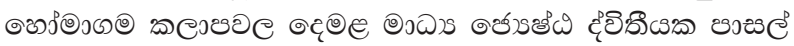

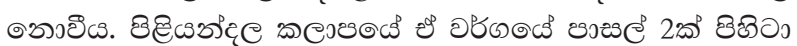

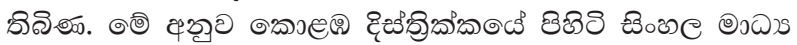

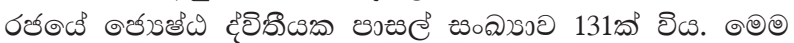

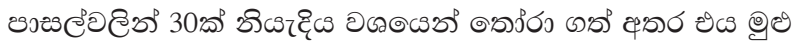

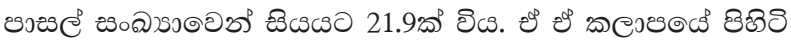

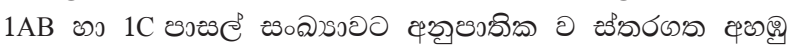
వరఅడ

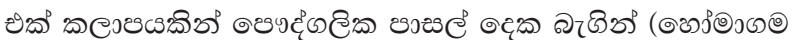

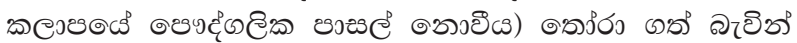

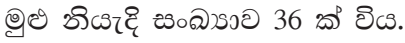

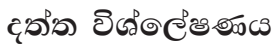

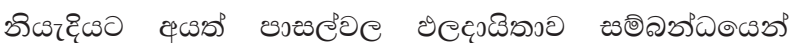

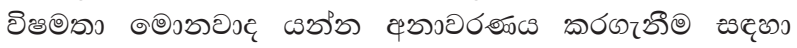

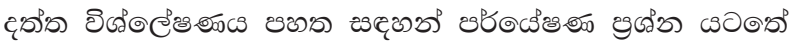

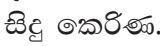

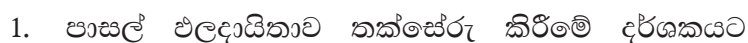

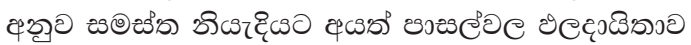

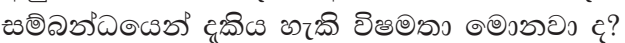

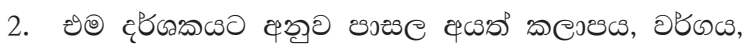

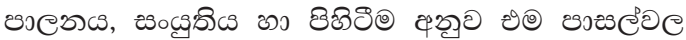

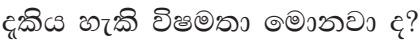

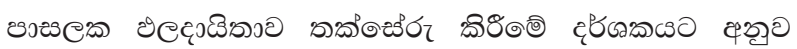

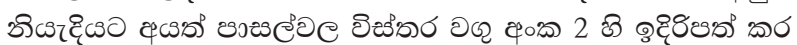

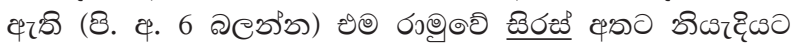

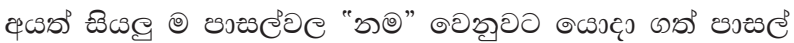

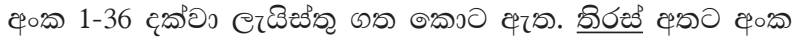

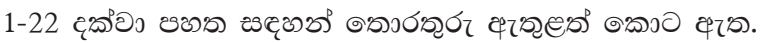

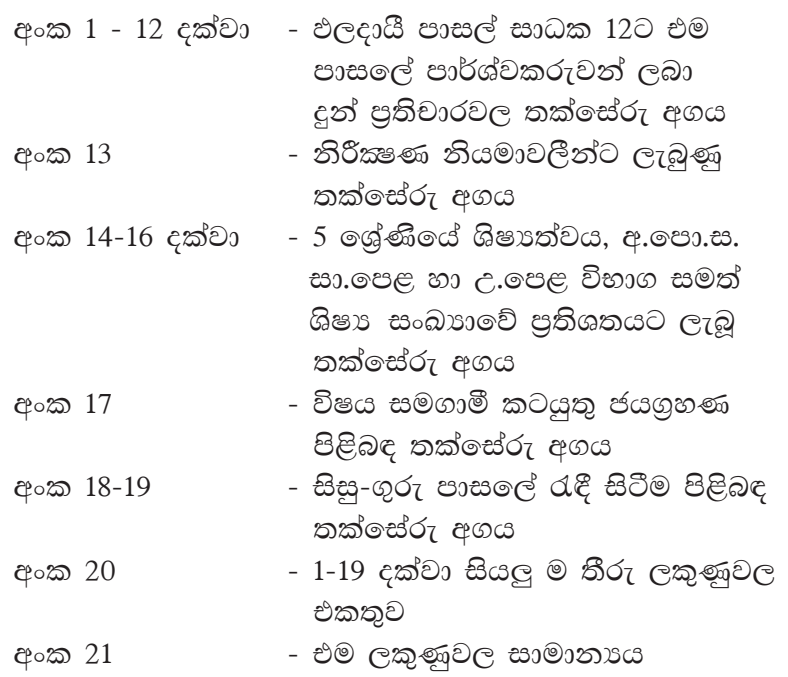

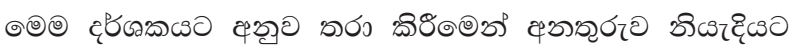

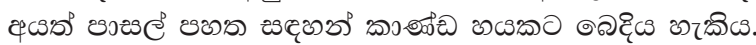

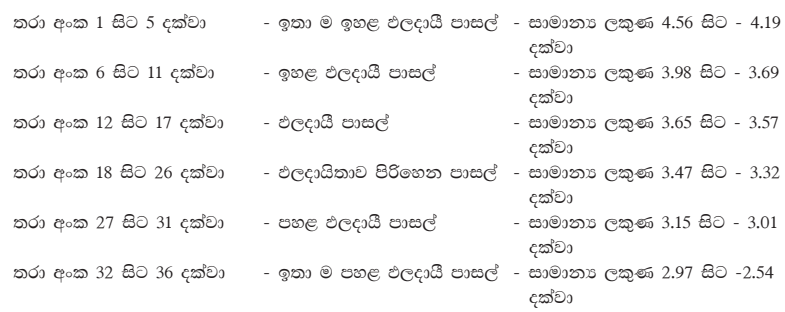

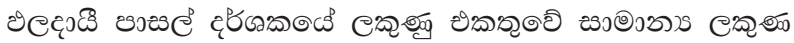

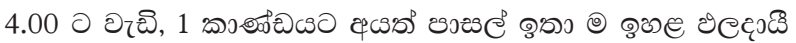

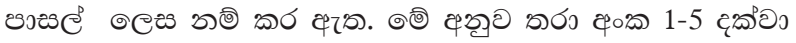

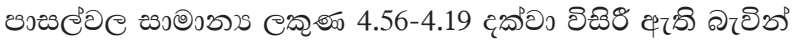

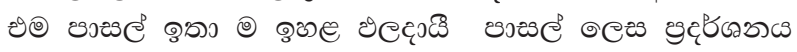

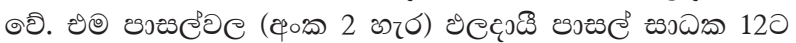

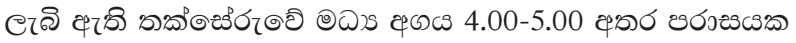

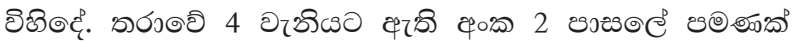

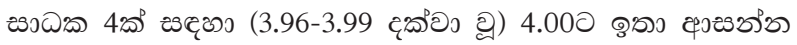

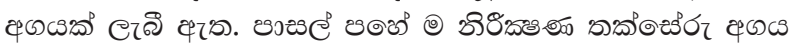

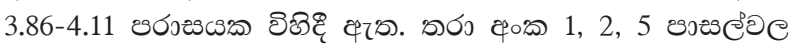

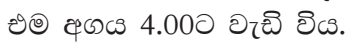

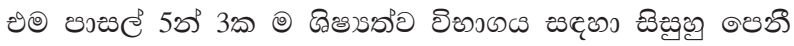

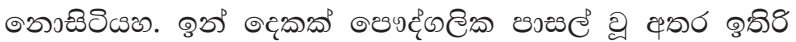

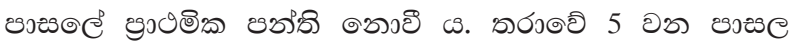

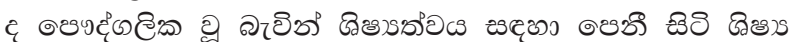

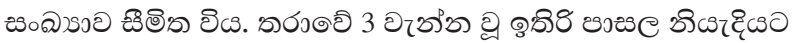

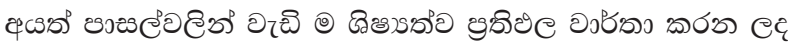

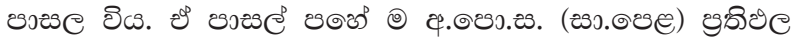

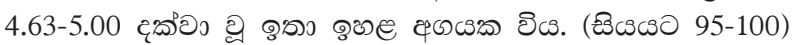

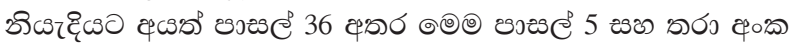

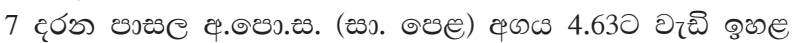

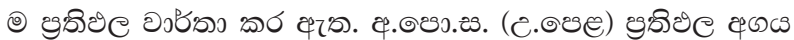

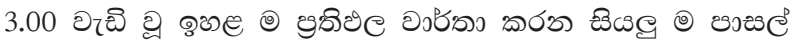

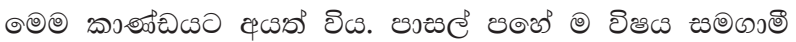

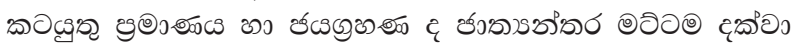

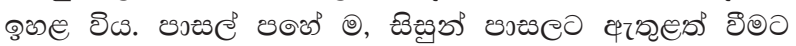

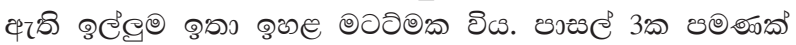

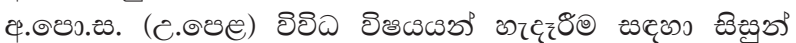

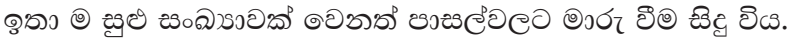

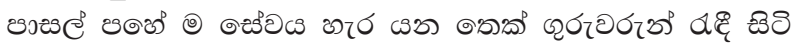

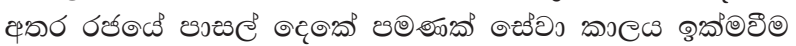

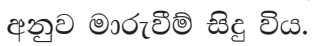

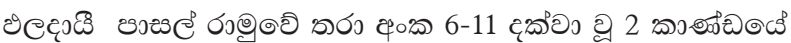

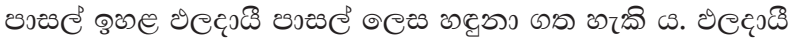

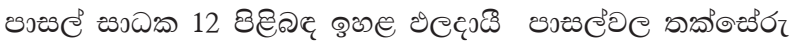

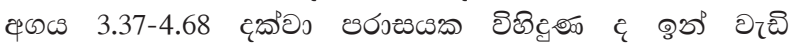

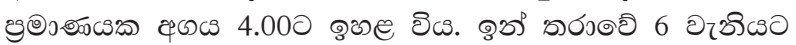

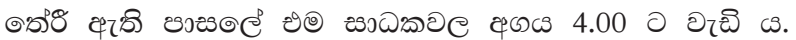

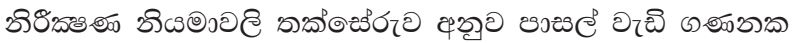

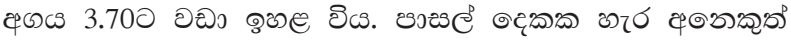




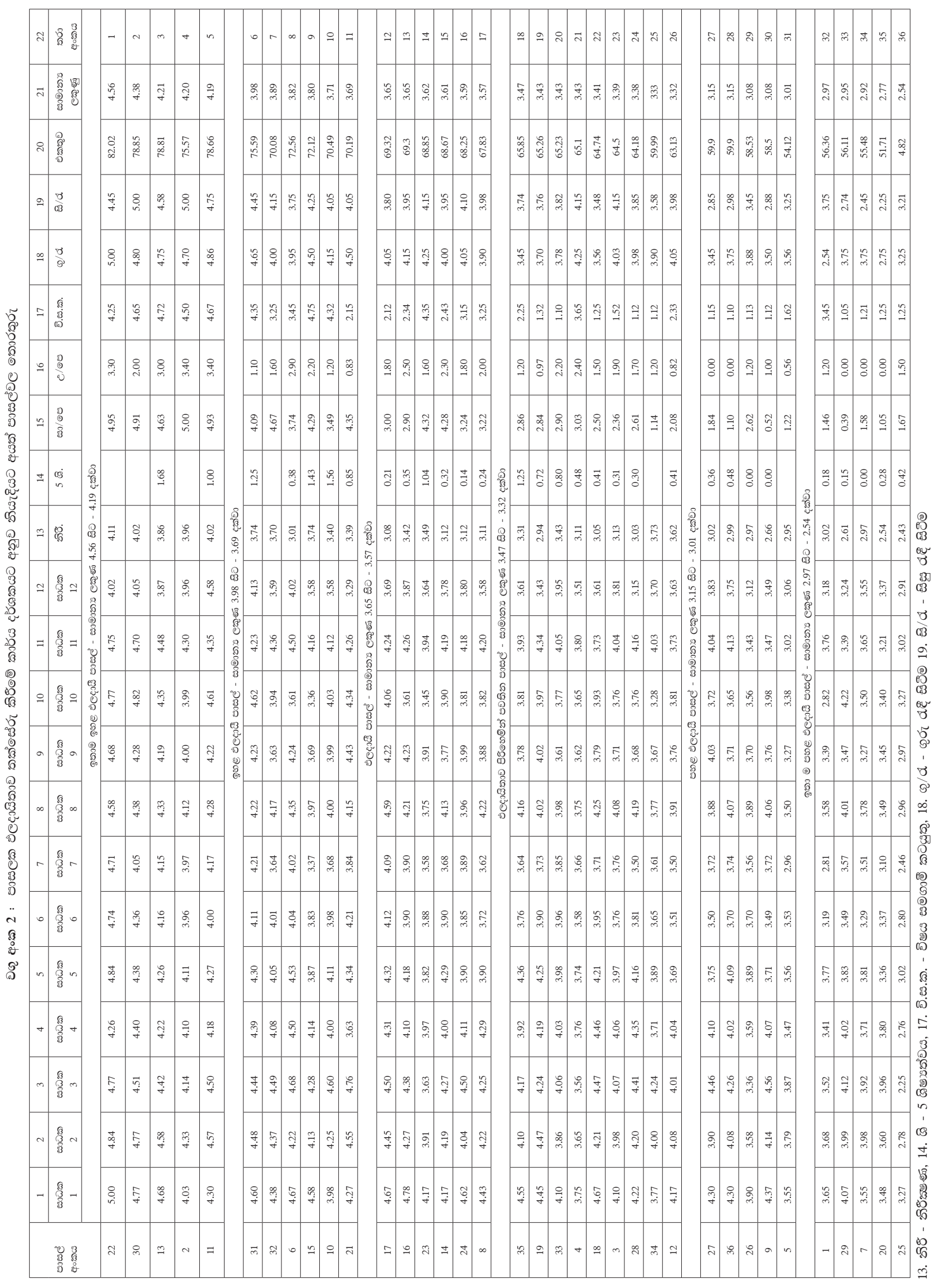




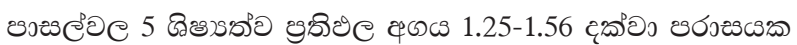

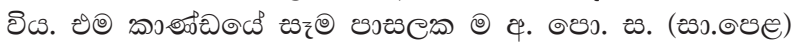

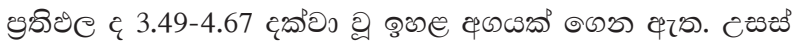

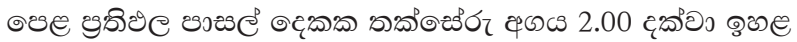

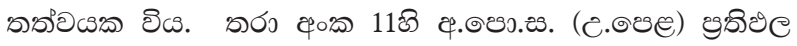

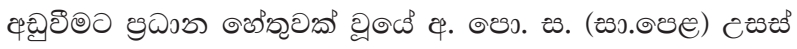

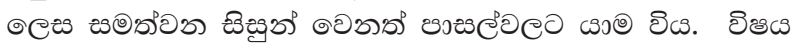

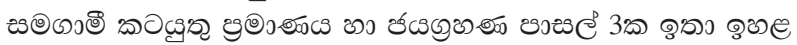

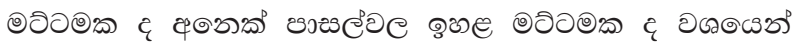

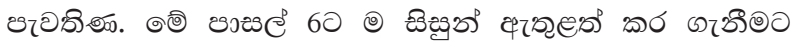

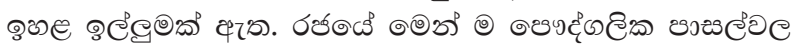

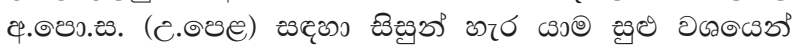

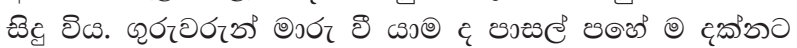

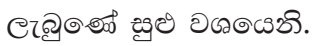

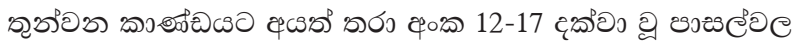

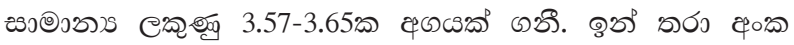

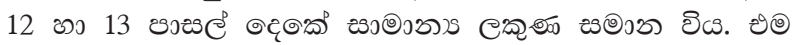

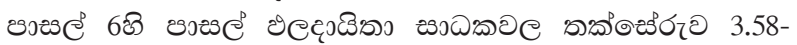

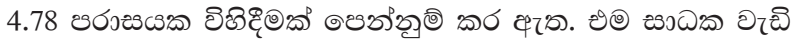

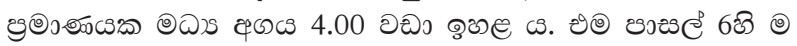

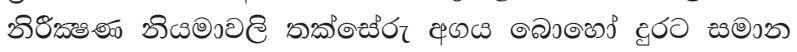

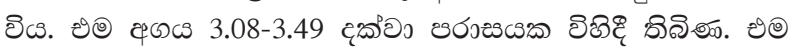

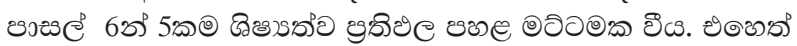

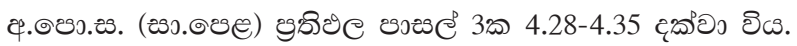

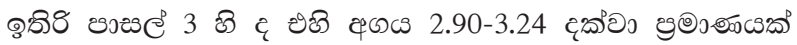

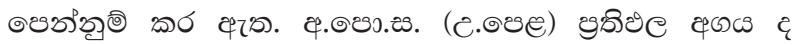

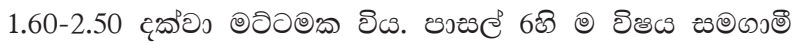

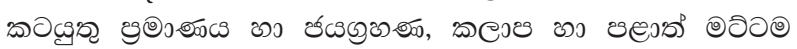

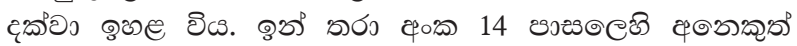

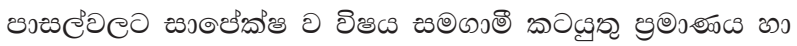

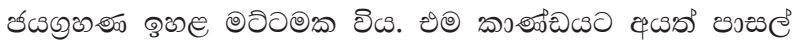

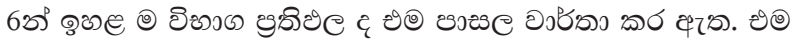

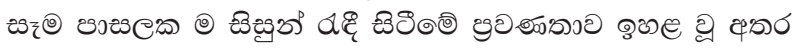

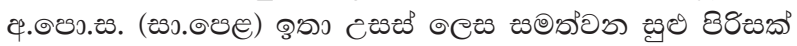

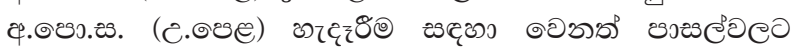

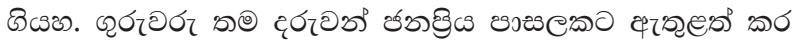

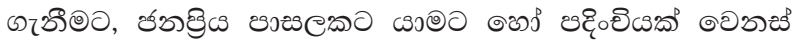

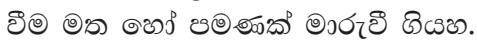

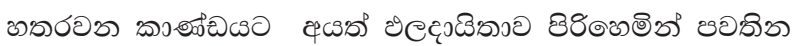

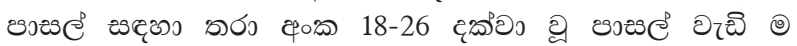

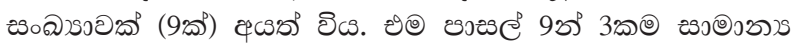

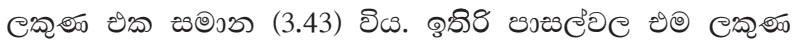

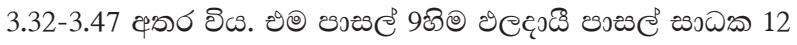

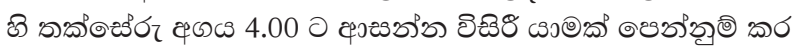

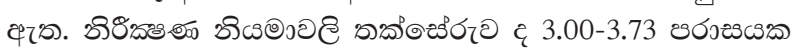

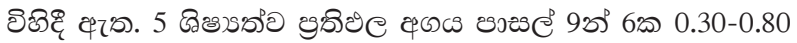

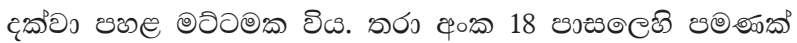

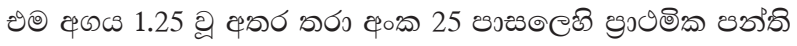

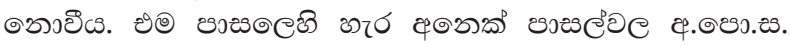

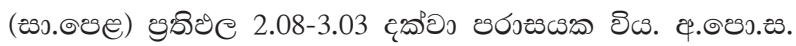

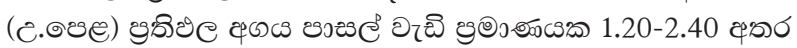

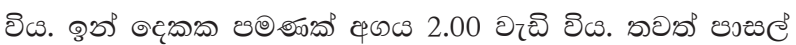

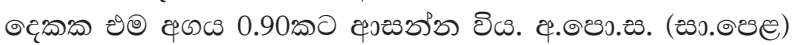

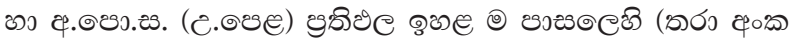

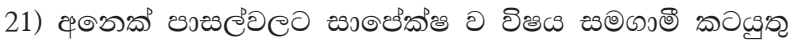

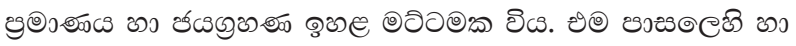

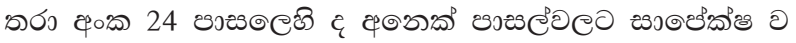

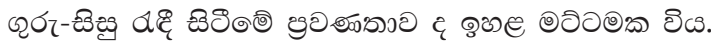

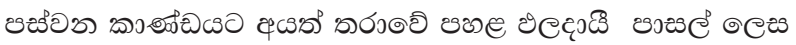

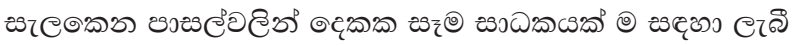

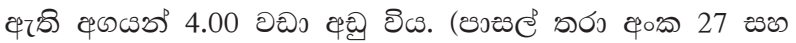

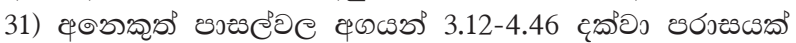

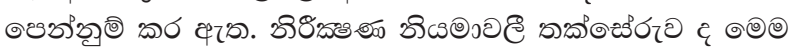

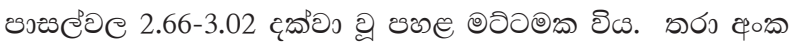

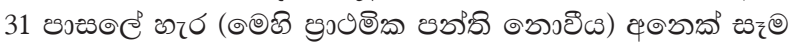

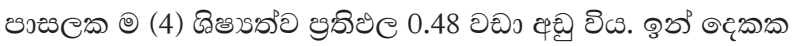

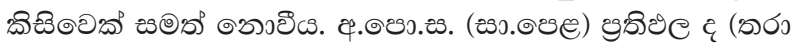

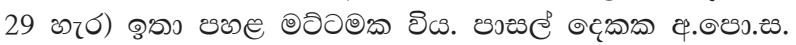

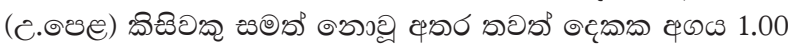

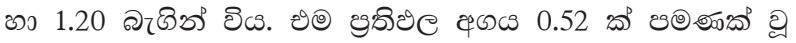

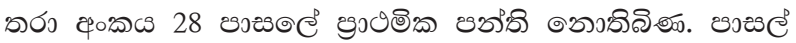

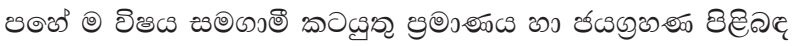

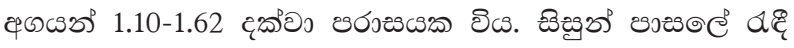

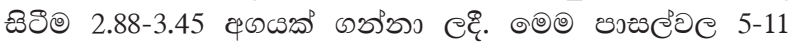

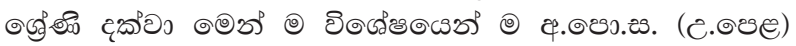

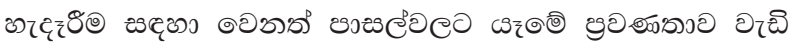

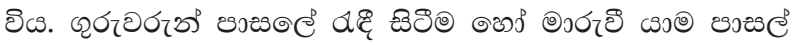

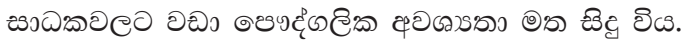

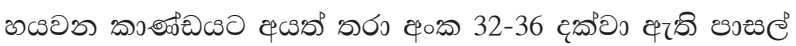

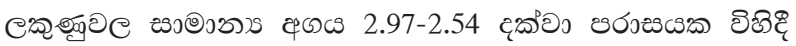

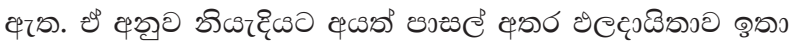

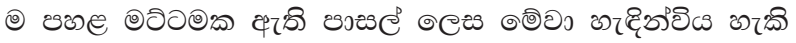

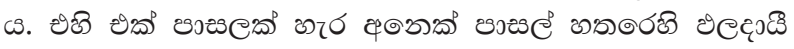

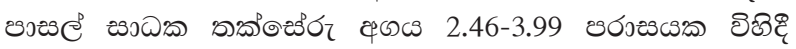

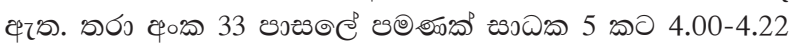

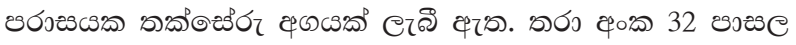

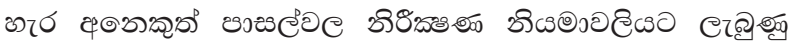

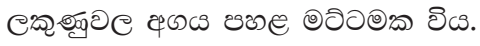

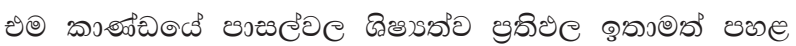

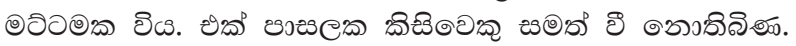

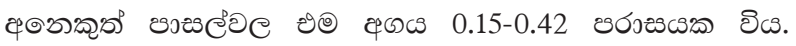

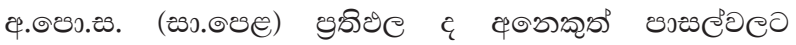

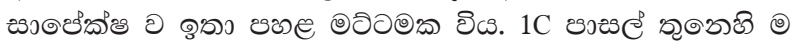

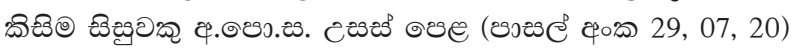

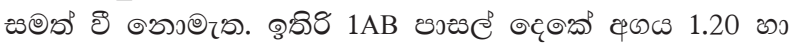

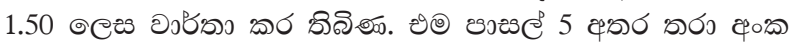

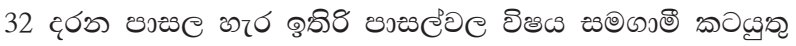

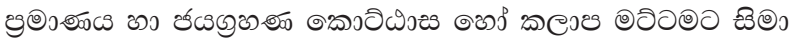

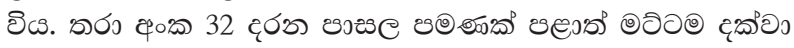

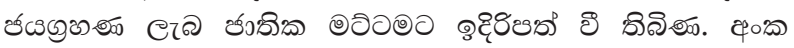

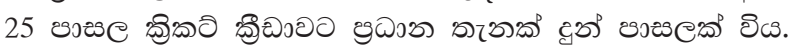




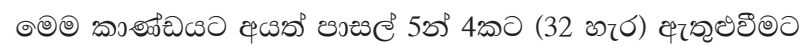

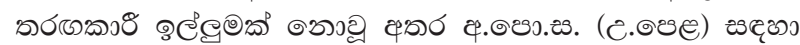

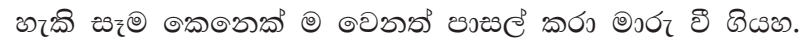

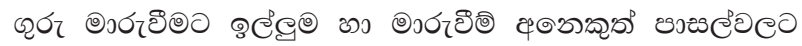

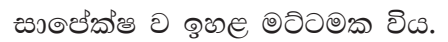

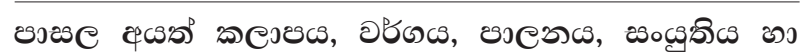

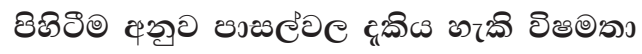

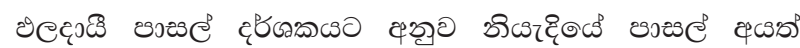

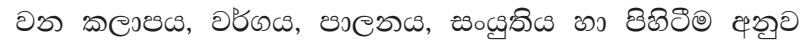

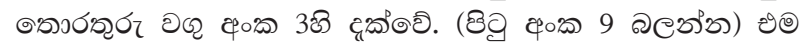

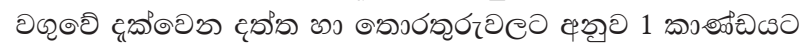

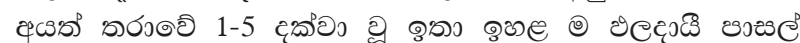

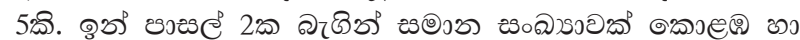

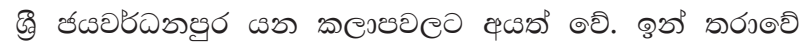

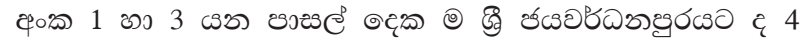

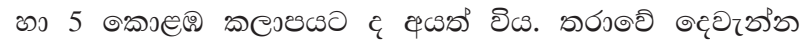

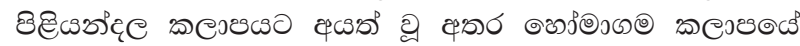

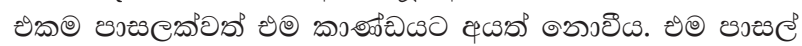

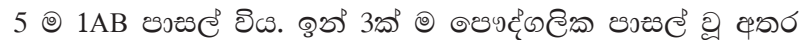

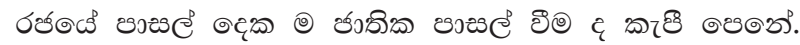

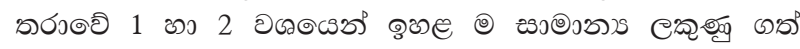

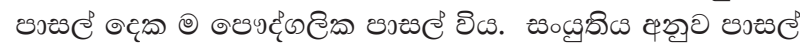

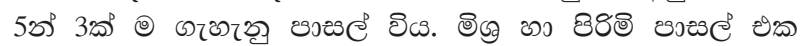

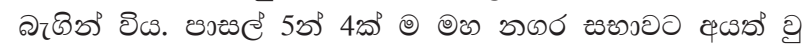

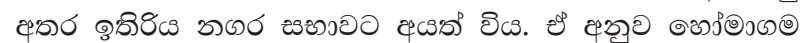

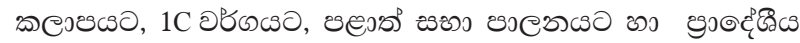

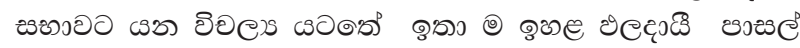

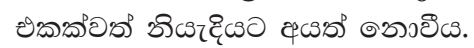

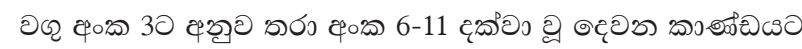

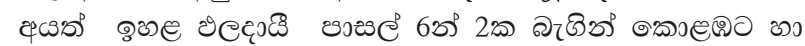

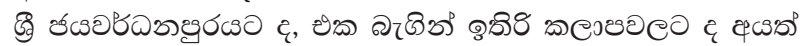

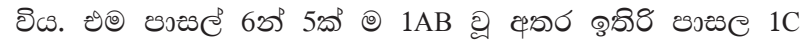

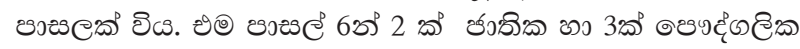

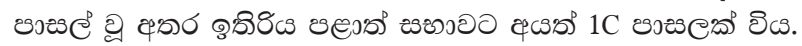

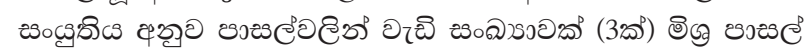

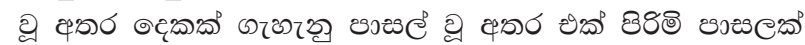

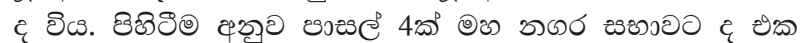

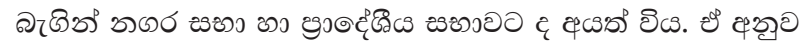

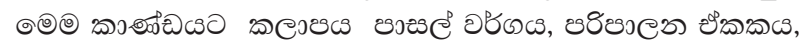

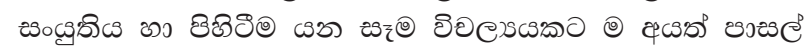

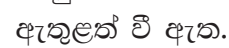

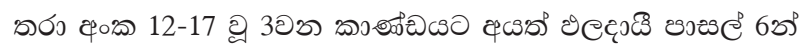

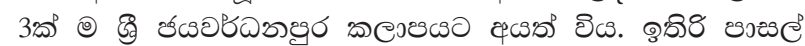
35 2

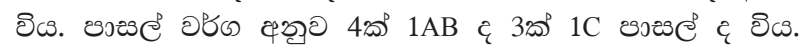

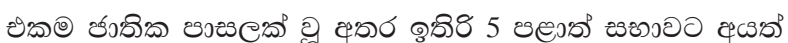

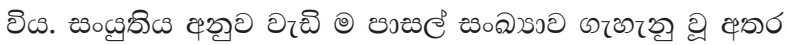

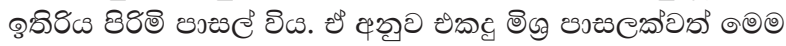

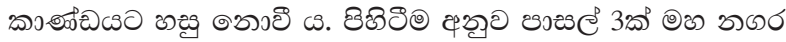

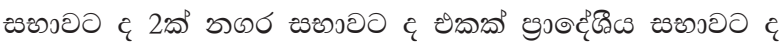
ү

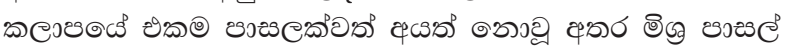

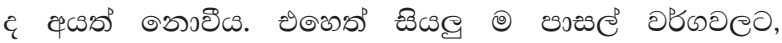

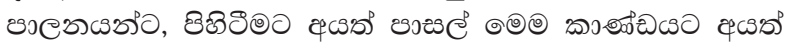
รื ริธิซ.

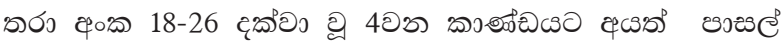

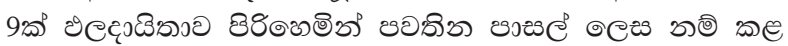

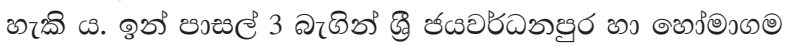

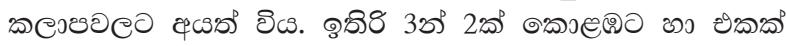

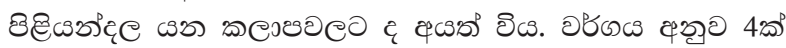

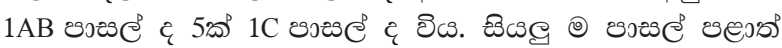

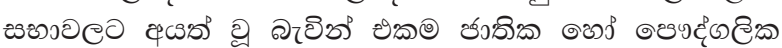

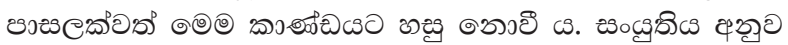

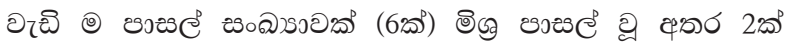

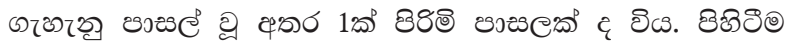

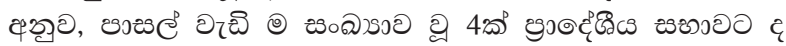

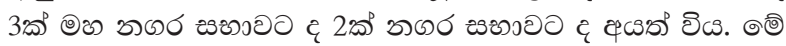

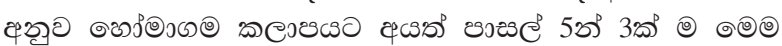

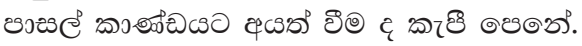

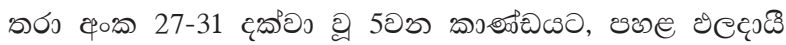

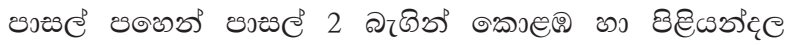

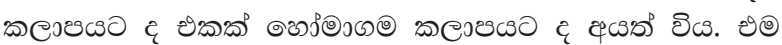
элщ

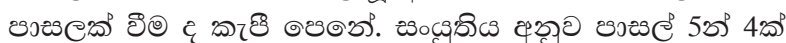

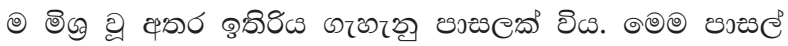

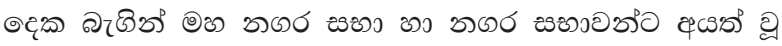

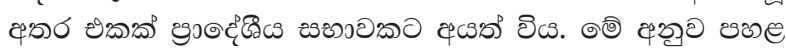

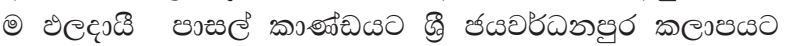

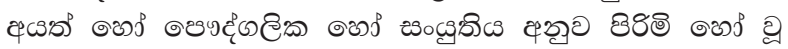

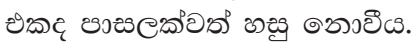

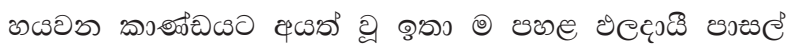

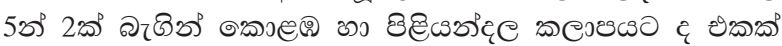

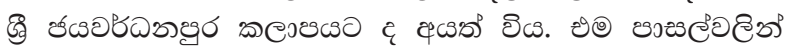

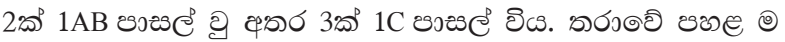
2 భం 36,1

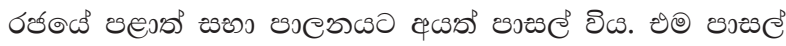

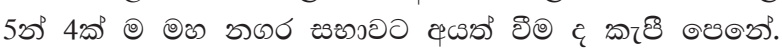

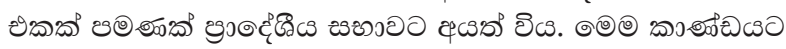

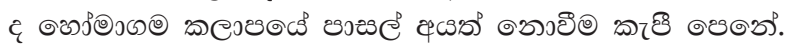

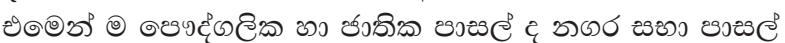

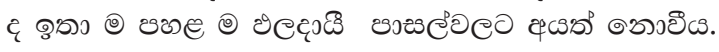




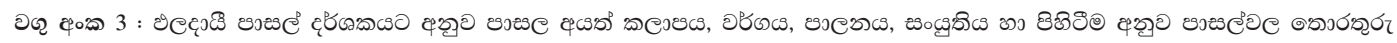

\begin{tabular}{|c|c|c|c|c|c|c|c|}
\hline 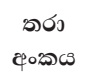 & జงองయว & 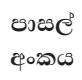 & meगతs & Dbscs & эеগ⿻ & 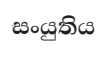 & తకరితితి \\
\hline 1 & 4.56 & 22 & శ్రి ోీอదదణత్రర & $1 \mathrm{AB}$ & ๑லэદ્द્ & తిర్య & ணைర జऽग \\
\hline 2 & 4.38 & 30 & 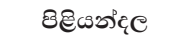 & $1 \mathrm{AB}$ & ๑லэદุ & ఆరతి & 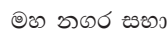 \\
\hline 3 & 4.21 & 13 & శ్రి ోడอదదణత్రం & $1 \mathrm{AB}$ & $\sigma / 3$ & 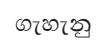 & 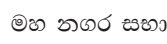 \\
\hline 4 & 4.21 & 02 & ๑2ుeఱ & $1 \mathrm{AB}$ & ర/శృ & 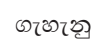 & (త) చుర జృ \\
\hline 5 & 4.19 & 11 & ๑வைలి & $1 \mathrm{AB}$ & ๑லэદุ & ஸてேてஒ & 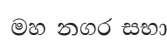 \\
\hline 6 & 3.98 & 31 & 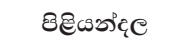 & $1 \mathrm{AB}$ & बওэद्ध & తిબ్రి & (อ) అుర జకు \\
\hline 7 & 3.89 & 32 & ๑ேรீอง๑ & $1 \mathrm{AB}$ & ర/శం & తిબ్య & 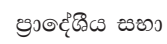 \\
\hline 8 & 3.82 & 06 & ๑2ుeอ & $1 \mathrm{C}$ & $\sigma / 3$ & 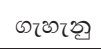 & (అ) చుర బఠు \\
\hline 9 & 3.80 & 15 & 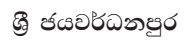 & $1 \mathrm{AB}$ & ర/శృ & తిర్య & అుర బళల \\
\hline 10 & 3.71 & 10 & ๑ฉులே & $1 \mathrm{AB}$ & ๑லэद्दे & ఆరఠి & (అ) కుర బఠు \\
\hline 11 & 3.69 & 21 & 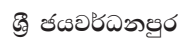 & $1 \mathrm{AB}$ & ๑Ъฯદ્ & ஸேோனุ & 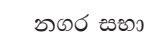 \\
\hline 12 & 3.65 & 17 & శ్రి శஸอళదตูర & $1 \mathrm{C}$ & $\sigma / \Theta$ & 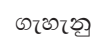 & అుర జరు \\
\hline 13 & 3.65 & 16 & 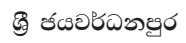 & $1 \mathrm{C}$ & $\sigma / 3$ & ఆ৪ఠి & 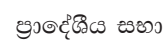 \\
\hline 14 & 3.62 & 23 & 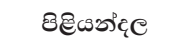 & $1 \mathrm{AB}$ & $\sigma / 3$ & ఆరఠతి & (అ) చుర జऽు \\
\hline 15 & 3.61 & 14 & శ్రి శஸอదదตูర & $1 \mathrm{AB}$ & $\sigma / ও$ & 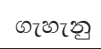 & అ๘ అుర జ \\
\hline 16 & 3.59 & 24 & 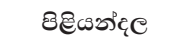 & $1 \mathrm{AB}$ & $\sigma / 3$ & 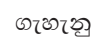 & (అ) అుర బవు \\
\hline 17 & 3.57 & 08 & ๑ロைe@ & $1 \mathrm{C}$ & $\sigma / 3$ & のろてつ & 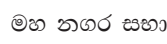 \\
\hline 18 & 3.47 & 35 & 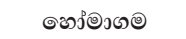 & $1 \mathrm{C}$ & $\sigma / 3$ & తిબ్య & 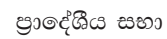 \\
\hline 19 & 3.43 & 19 & 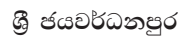 & $1 \mathrm{C}$ & $\sigma / 8$ & తిర్త & అుర జலు \\
\hline 20 & 3.43 & 33 & 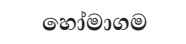 & $1 \mathrm{AB}$ & $\sigma / 3$ & తిర్తి & 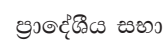 \\
\hline 21 & 3.43 & 04 & ๑2ைలఱ & $1 \mathrm{AB}$ & $\sigma / 3$ & తిબ్య & (అ) చుర జిం \\
\hline 22 & 3.41 & 18 & 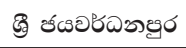 & $1 \mathrm{C}$ & $\sigma / 3$ & తియ్త & అుర జి \\
\hline 23 & 3.39 & 03 & ๑ロைe@ & $1 \mathrm{AB}$ & $\sigma / 3$ & 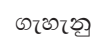 & 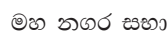 \\
\hline 24 & 3.38 & 28 & 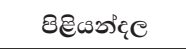 & $1 \mathrm{C}$ & $\sigma / \diamond$ & 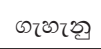 & 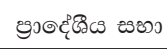 \\
\hline 25 & 3.33 & 34 & ๑ேวீองตอ & $1 \mathrm{C}$ & $\sigma / 3$ & తిબ్య & 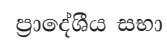 \\
\hline 26 & 3.32 & 12 & 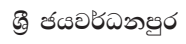 & $1 \mathrm{AB}$ & $\sigma / \Theta$ & ఈ৪తి & అ๘ అుర జ \\
\hline 27 & 3.15 & 27 & 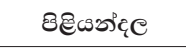 & $1 \mathrm{C}$ & $\sigma / 3$ & తిర్త & ऊుర జయ \\
\hline 28 & 3.15 & 36 & ๑ふวீ๑งอ & $1 \mathrm{C}$ & $\sigma / 3$ & తిબ్య & 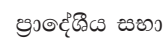 \\
\hline 29 & 3.08 & 26 & 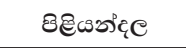 & $1 \mathrm{C}$ & $\sigma / \Theta$ & બてひてð & అుర బலు \\
\hline 30 & 3.08 & 09 & ๑ロుe@ & $1 \mathrm{C}$ & $\sigma / 3$ & తిర్య & 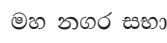 \\
\hline 31 & 3.01 & 05 & ๑๐ుe@ & $1 \mathrm{AB}$ & ర/శృ & తిબ్త & (అ) చుర జకు \\
\hline 32 & 2.97 & 01 & ๑ேைย & $1 \mathrm{AB}$ & $\sigma / 8$ & ఆరఠి & అఅ అుర జకు \\
\hline 33 & 2.95 & 29 & 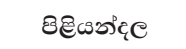 & $1 \mathrm{C}$ & $\sigma / 3$ & ఆరఠ & అ๘ అుర జઝ \\
\hline 34 & 2.92 & 07 & ๑ロుe® & $1 \mathrm{C}$ & $\sigma / \Theta$ & ఆరఠి & 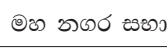 \\
\hline 35 & 2.77 & 20 & శ్రి ోตอరదణత్రం & $1 \mathrm{C}$ & $\sigma / 3$ & తిબ్య & 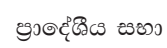 \\
\hline 36 & 2.54 & 25 & 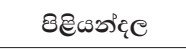 & $1 \mathrm{AB}$ & $\sigma / \Theta$ & తొర్తి & 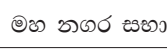 \\
\hline
\end{tabular}

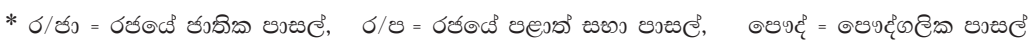




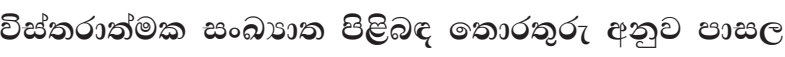

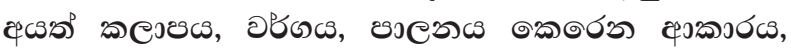

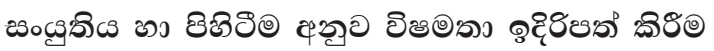

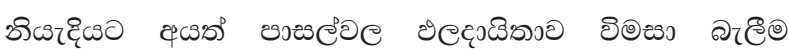

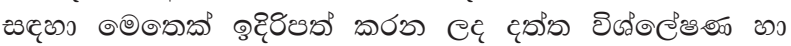

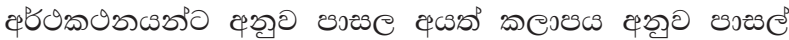

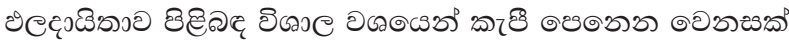

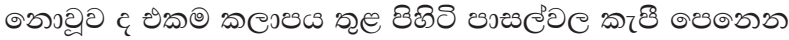

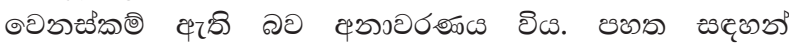
రిజ

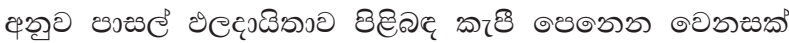

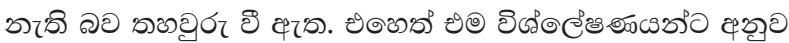

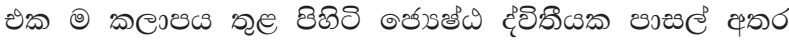

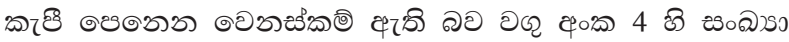

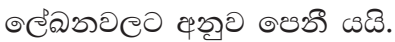

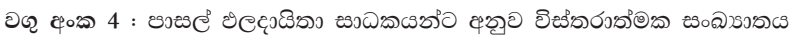

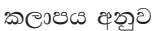

\begin{tabular}{|c|c|c|c|c|c|c|}
\hline \multirow{2}{*}{ пелесs } & \multirow{2}{*}{ 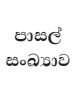 } & \multicolumn{5}{|c|}{ 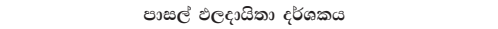 } \\
\hline & & $\begin{array}{l}\text { \%еอ } \\
\text { \%ow }\end{array}$ & 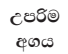 & बอூద & ews & 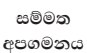 \\
\hline ๑ேைе & 11 & 2.76 & 4.26 & 1.50 & 3.42 & 0.4464 \\
\hline 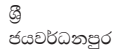 & 11 & 3.01 & 4.70 & 1.69 & 3.67 & 0.4443 \\
\hline 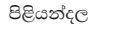 & 09 & 2.51 & 4.46 & 1.95 & 3.41 & 0.5723 \\
\hline ๑๐ீอேอ & 05 & 3.27 & 4.16 & 0.89 & 3.59 & 0.3390 \\
\hline
\end{tabular}

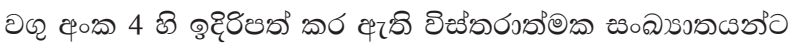

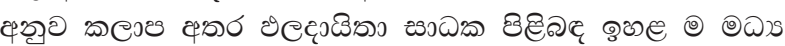

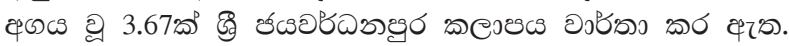

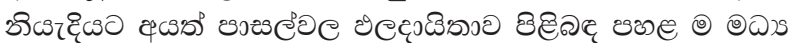

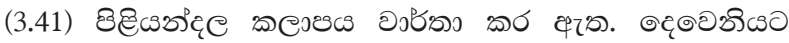

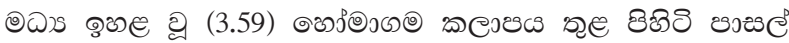

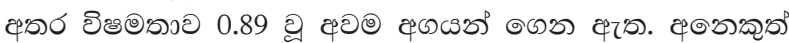
๓елюอе

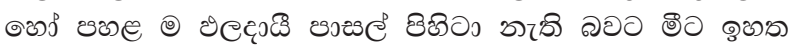

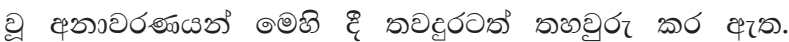

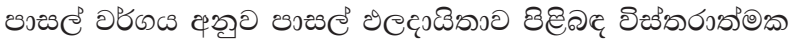

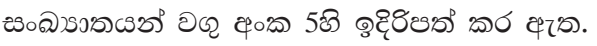

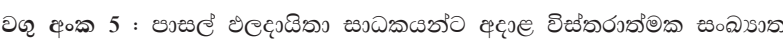

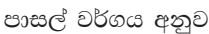

\begin{tabular}{|c|c|c|c|c|c|c|}
\hline \multirow[b]{2}{*}{ 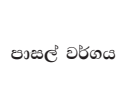 } & \multirow{2}{*}{ 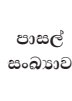 } & \multicolumn{5}{|c|}{ 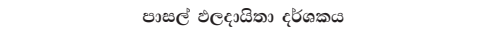 } \\
\hline & & $\begin{array}{l}\text { ¿еల } \\
\text { cow }\end{array}$ & $\begin{array}{l}\text { cosరอ } \\
\text { कृतs }\end{array}$ & ๑อిజ & ๑๐ & 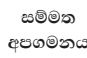 \\
\hline $1 \mathrm{AB}$ & 20 & 2.51 & 4.70 & 2.19 & 3.67 & 0.5447 \\
\hline $1 \mathrm{C}$ & 16 & 2.87 & 3.72 & 0.85 & 3.33 & 0.2626 \\
\hline
\end{tabular}

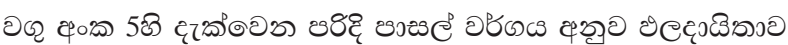

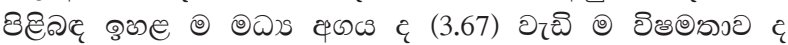

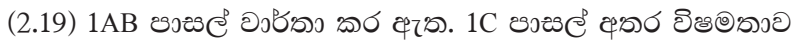

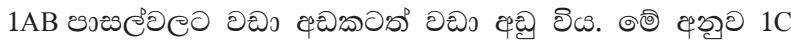

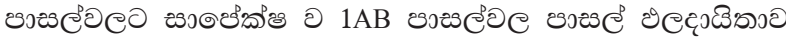

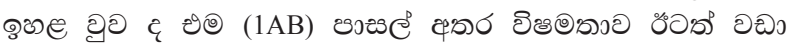

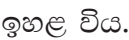

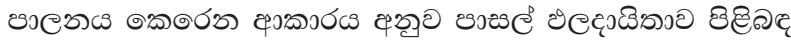

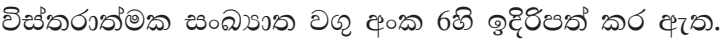

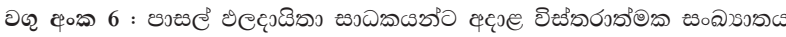

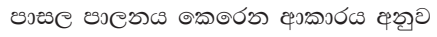

\begin{tabular}{|c|c|c|c|c|c|c|}
\hline \multirow[b]{2}{*}{ sowe อbow } & \multirow{2}{*}{ 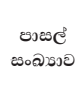 } & \multicolumn{5}{|c|}{ 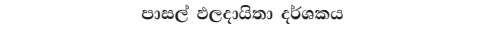 } \\
\hline & & $\begin{array}{l}\text { \%eอ } \\
\text { cons }\end{array}$ & 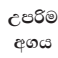 & बอజ & ๑D:s & 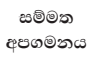 \\
\hline 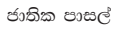 & 06 & 3.11 & 4.26 & 1.15 & 3.84 & 0.4318 \\
\hline 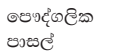 & 06 & 3.72 & 4.70 & 0.98 & 4.09 & 0.3920 \\
\hline 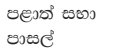 & 24 & 2.51 & 3.72 & 1.21 & 3.29 & 0.3018 \\
\hline
\end{tabular}

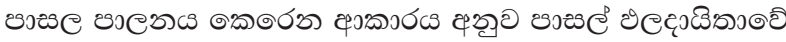

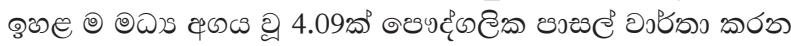

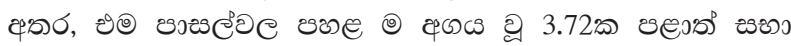

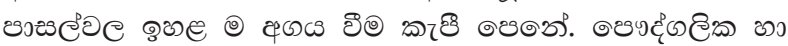

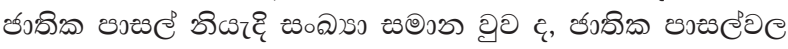

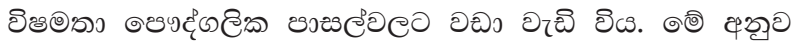

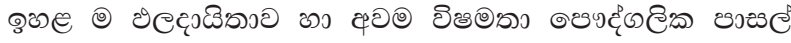

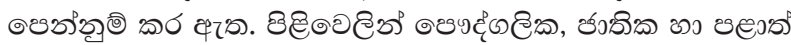

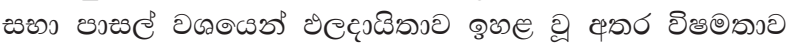
दุ ๕ออ ฮิต.

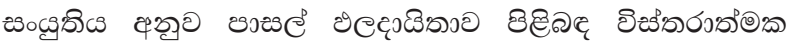

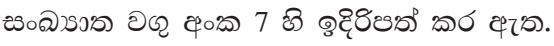

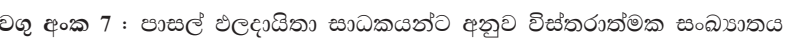

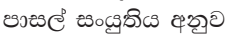

\begin{tabular}{|c|c|c|c|c|c|c|}
\hline \multirow[b]{2}{*}{ 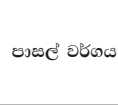 } & \multirow{2}{*}{$\begin{array}{c}\text { जsese } \\
\text { woDsse }\end{array}$} & \multicolumn{5}{|c|}{ 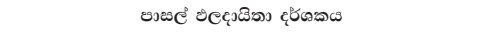 } \\
\hline & & $\begin{array}{l}\text { \%еల } \\
\text { cocs }\end{array}$ & 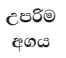 & - Dோ & ๑ڤs & 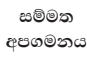 \\
\hline తిరి & 8 & 2.76 & 4.46 & 1.70 & 3.40 & 0.5606 \\
\hline の) & 12 & 3.16 & 4.26 & 1.10 & 3.64 & 0.3420 \\
\hline తిఱ్య & 16 & 2.51 & 4.70 & 2.19 & 3.47 & 0.5092 \\
\hline
\end{tabular}

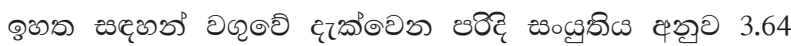

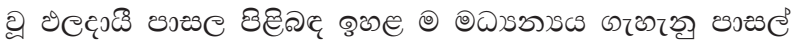

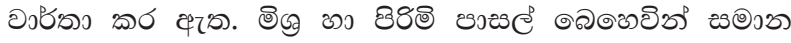

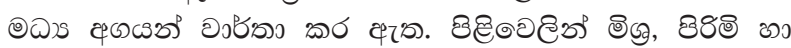

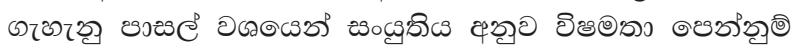

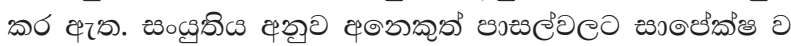

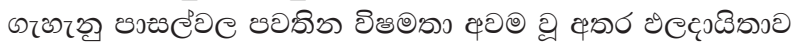
๑ே) లిఱ. 


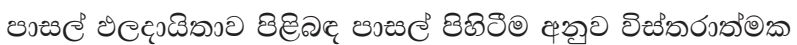

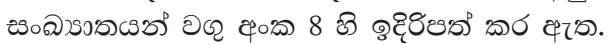

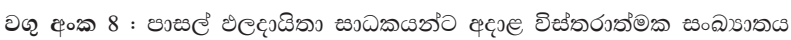

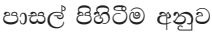

\begin{tabular}{|c|c|c|c|c|c|c|}
\hline \multirow[b]{2}{*}{ 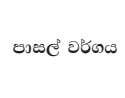 } & \multirow{2}{*}{ 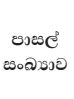 } & \multicolumn{5}{|c|}{ 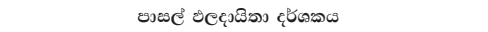 } \\
\hline & & $\begin{array}{l}\text { ве० } \\
\text { вos }\end{array}$ & $\begin{array}{l}\text { cose } \\
\text { enc }\end{array}$ & - Dases & ๑ळ) & 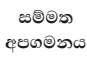 \\
\hline అయ అుర జాలు & 19 & 2.51 & 4.46 & 1.95 & 3.46 & 0.5336 \\
\hline 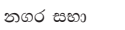 & 07 & 3.46 & 4.70 & 1.24 & 3.78 & 0.4228 \\
\hline 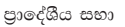 & 10 & 3.01 & 4.16 & 1.15 & 3.42 & 0.3121 \\
\hline
\end{tabular}

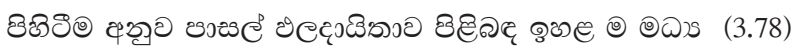

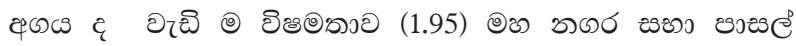

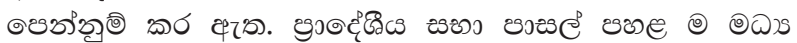

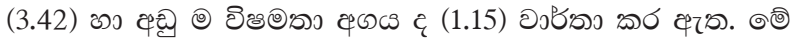

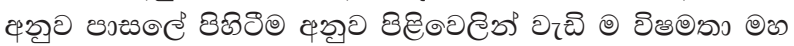

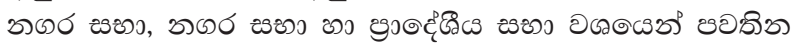

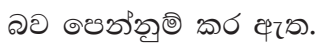

\section{ริตอุร}

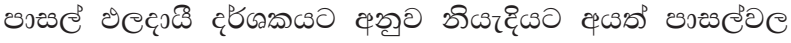

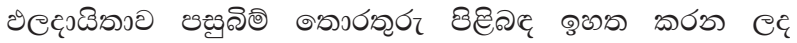

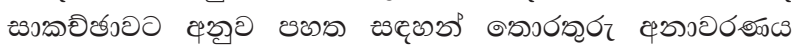
లిต.

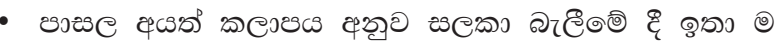

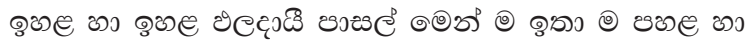

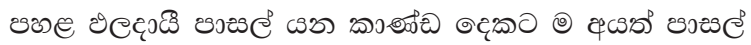

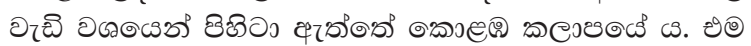

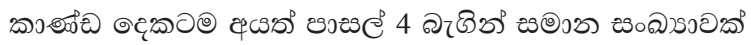

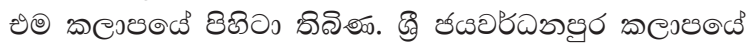

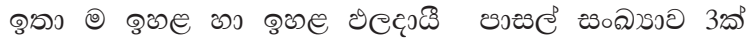

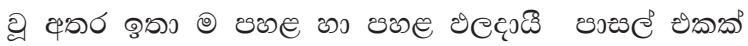

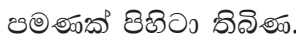

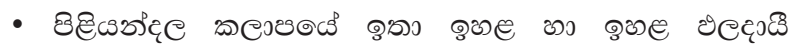

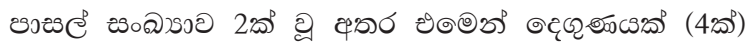

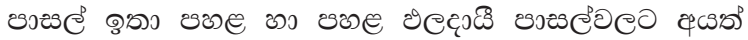

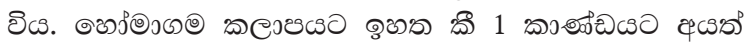

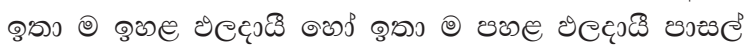

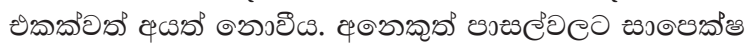

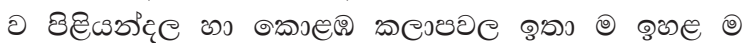

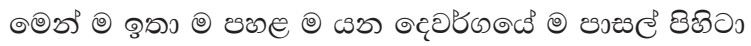

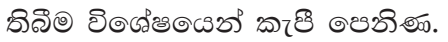

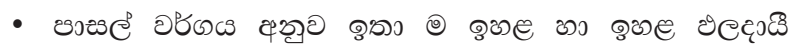

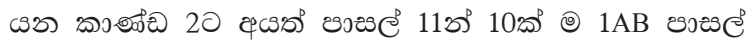

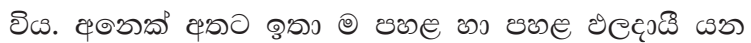

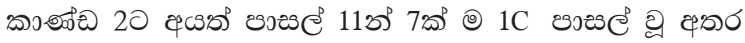

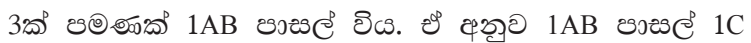

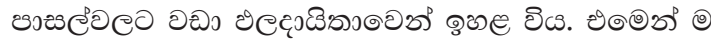

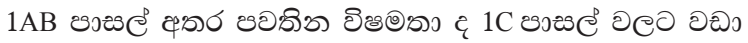
๑Ъల రిడ.

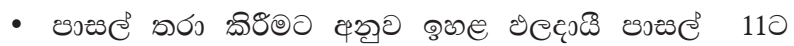

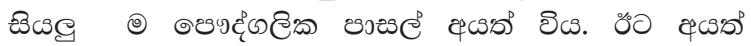

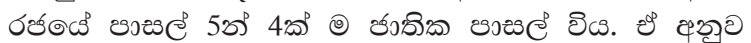

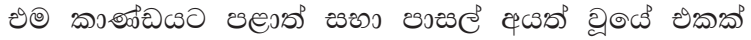

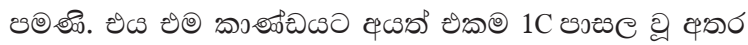

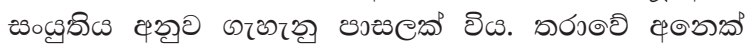

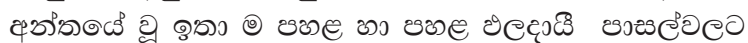

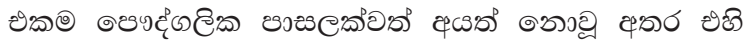

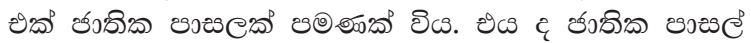

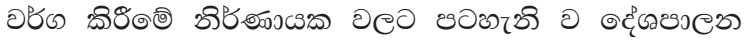

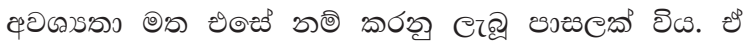

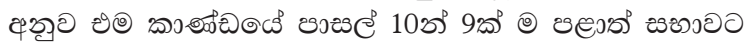
భ

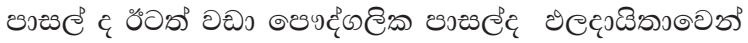
ஓ๘) כิఱ.

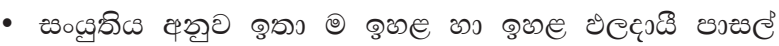

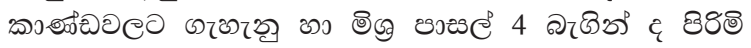

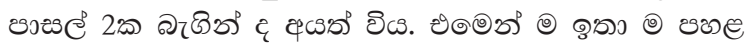

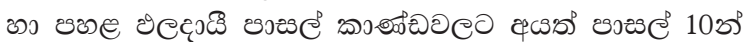

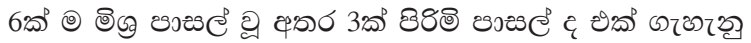

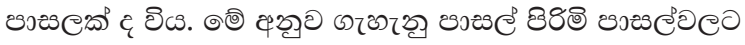

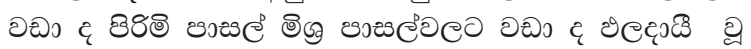

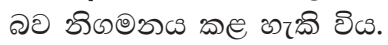

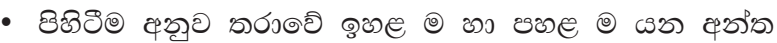

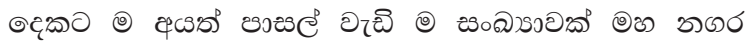

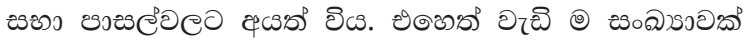

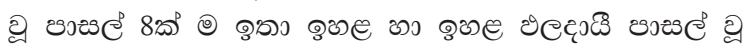
ॠ

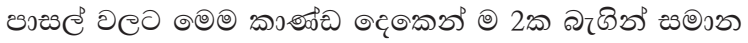

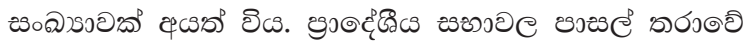

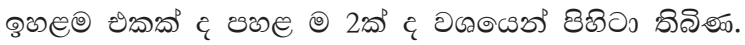

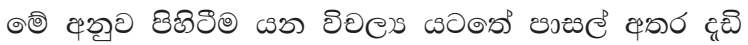

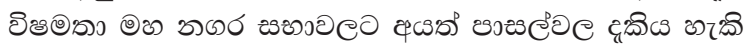

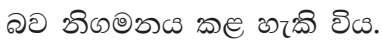

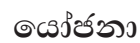

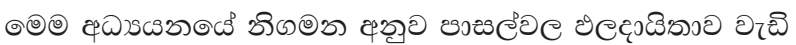

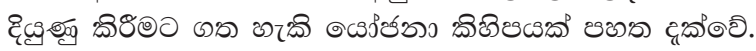

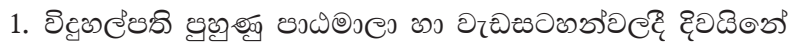

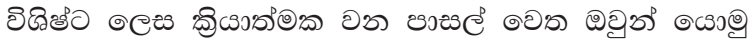

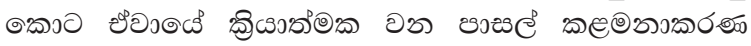

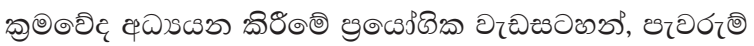

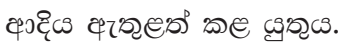




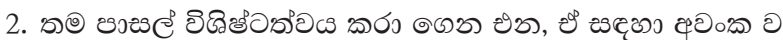

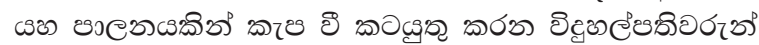

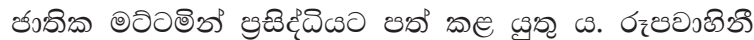

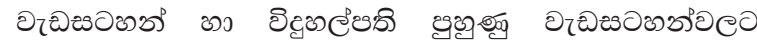

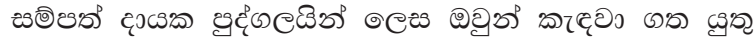

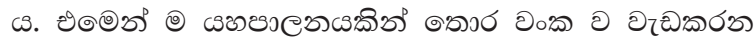

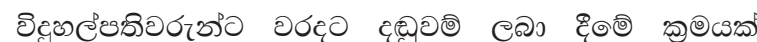

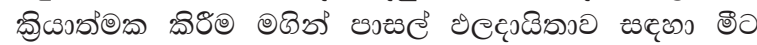

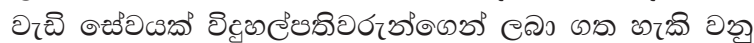
๘๐ุ.

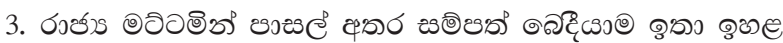

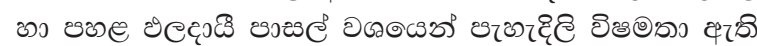

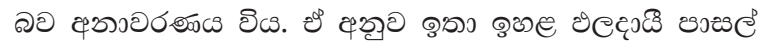

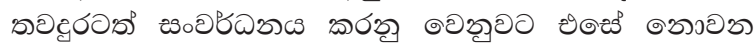

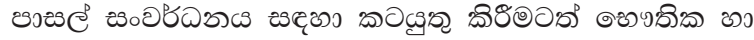

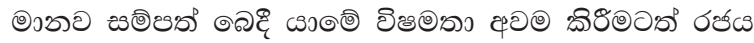

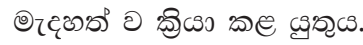

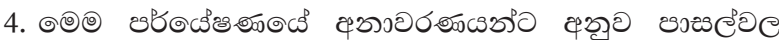

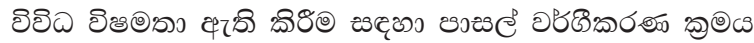

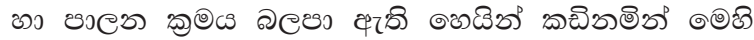

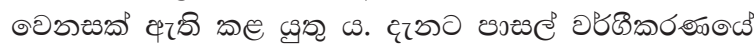

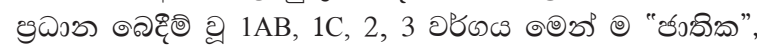

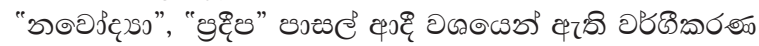

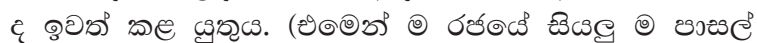

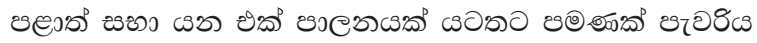
(ุ⿹勹冫 (S)

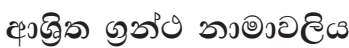

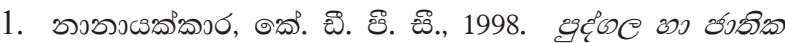

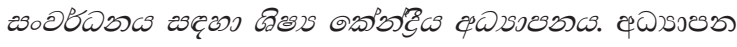

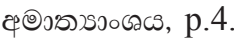

2. Austin, G., 1979. Exemplary Schools \& the Search for Effectiveness. Educational Leadership, 37 (1), pp. $10-4$.

3. Baker, V.J., 1988. The Blackboard in the Jungle: Formal Education in Disadvantaged Rural Areas. Netherlands: Eburoh Publishers.
4. Chapman, J., 1990. The Effectiveness of Schooling and of Educational Resource Management. Paris: Paper Prepared for OECD.

5. Gafoor, K.A. \& Faroogue, T.K., 2006. Comparison of High and Low Efficient Schools in Terms of School, Pupil and Home - Related Variables, Journal of Indian Education, 32.

6. Levin, H. \& Lockheed, R., 1993. Effective Schools in Developing Countries. London: Palmer Press.

7. Look, D., 2006. Defining Effective Schools, Discussion Paper - Prepared for PUSD Excellence Committee, US Federal Government Excellence Committee, International Journal of Educational Development, 28 (6), pp. 669- 86.

8. McGraw, B. \& Piper, K., 1992. The Effective Schools Project. In: B. McGraw, K. Piper, D.Banks and B. Evans. Making Schools More Effective. Hawthorn: Australian Council for Educational Research. Melbourne, Australia.

9. Mellor, W. \& Chapman, J., 1984. Organizational Effectiveness in Schools. Education Administration Review, 2 (2), pp. $25-36$.

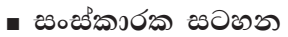

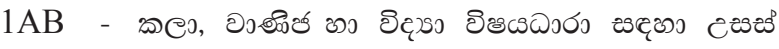

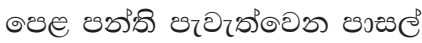

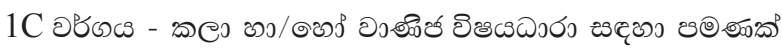

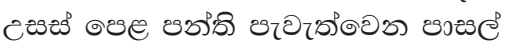

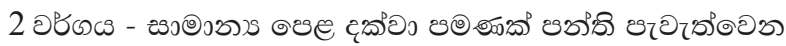
วงะือ

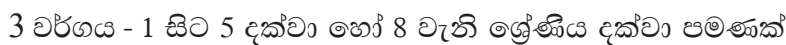

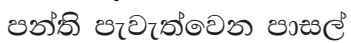

